\title{
Data Collection versus Data Estimation: A Fundamental Trade-off in Dynamic Networks
}

\author{
Jalal Arabneydi, Member, IEEE, and Amir G. Aghdam, Senior Member, IEEE
}

\begin{abstract}
An important question that often arises in the operation of networked systems is whether to collect the realtime data or to estimate them based on the previously collected data. Various factors should be taken into account such as how informative the data are at each time instant for state estimation, how costly and credible the collected data are, and how rapidly the data vary with time. The above question can be formulated as a dynamic decision making problem with imperfect information structure, where a decision maker wishes to find an efficient way to switch between data collection and data estimation while the quality of the estimation depends on the previously collected data (i.e., duality effect). In this paper, the evolution of the state of each node is modeled as an exchangeable Markov process for discrete features and equivariant linear system for continuous features, where the data of interest are defined in the former case as the empirical distribution of the states, and in the latter case as the weighted average of the states. When the data are collected, they may or may not be credible, according to a Bernoulli distribution. Based on a novel planning space, a Bellman equation is proposed to identify a near-optimal strategy whose computational complexity is logarithmic with respect to the inverse of the desired maximum distance from the optimal solution, and polynomial with respect to the number of nodes. A reinforcement learning algorithm is developed for the case when the model is not known exactly, and its convergence to the nearoptimal solution is shown subsequently. In addition, a certainty threshold is introduced that determines when data estimation is more desirable than data collection, as the number of nodes increases. For the special case of linear dynamics, a separation principle is constructed wherein the optimal estimate is computed by a Kalman-like filter, irrespective of the probability distribution of random variables. It is shown that the complexity of finding the proposed sampling strategy, in this special case, is independent of the size of the state space and the number of nodes. Examples of a sensor network, a communication network and a social network are provided.
\end{abstract}

Index Terms-Networked systems, Partially observable Markov decision process, reinforcement learning, separation principle.

\section{INTRODUCTION}

The trade-off between the cost and value of information emerges in different types of networks, where it is important to monitor the status of the network by analyzing its real-time data. There is often a cost associated with collecting the data;

This work has been supported in part by Natural Sciences and Engineering Research Council of Canada (NSERC) under Grant RGPIN-262127-17, and in part by Concordia University under Horizon Postdoctoral Fellowship.

The authors are with Department of Electrical and Computer Engineering, Concordia University, 1455 de Maisonneuve Blvd, Montreal, QC, Canada. Email:jalal.arabneydi@mail.mcgill.ca and Email : aghdameece.concordia.ca

This article has supplementary downloadable material available at http: / / ieeexplore.ieee.org provided by the authors.

Digital Object Identifier 10.1109/TSNE.2020.2966504 hence, the question arises as when an estimate of the network data would be more desirable than monitoring the operation of the network.

A partially observable Markov decision process (POMDP) model is presented in this paper to address the above tradeoff in invariant/equivariant networks. The data are defined as the empirical distribution and weighted average of the states of the nodes for finite and infinite state spaces, respectively, and the per-step cost function incorporates the two competing concepts of the above trade-off: the cost of information and the value of information. It is to be noted that the empirical distribution and weighted average (i.e., linear regression) of states are the data of interest in various applications, specially the ones modelled by deep neural networks that have recently received much attention [1, 2]. For example, the empirical distribution appears in Markov-chain deep teams wherein the nodes are partitioned into several sub-populations such that the system is invariant to the permutation of nodes in each sub-population [3]. The weighted average, on the other hand, emerges in linear quadratic deep teams wherein the nodes in each sub-population are not necessarily exchangeable and can have different weights [4]. See other examples in social networks [5], epidemics [6], cyber-security networks [7] and smart grids [8], to name only a few.

In general, finding the optimal solution of an infinite-horizon discounted cost POMDP is an undecidable problem [9] 1 and even finding a near-optimal solution is NP-hard [11]. On the other hand, it is not always possible to have sufficiently accurate knowledge of the model of nodes. Thus, it is important to learn the solution when the exact model is not available, and this clearly adds to the complexity of the problem. Due to the complexity of finding a near-optimal solution, most of the existing work is mainly focused on developing approximation methods for POMDPs, some of which are briefly reviewed in the next paragraph.

In grid-based approaches, an approximate value function is computed at a fixed number of points (called grids), and then interpolated over the entire belief space [12]. The advantage of such approaches is that their computational complexity remains the same at every iteration (i.e., does not increase with time) but their drawback is that the fixed points may not be reachable. In point-based methods, the reachability problem is addressed by considering the reachable set only, where an approximate value function is calculated iteratively

\footnotetext{
${ }^{1}$ The computational complexity of finding the optimal solution of the finite-horizon POMDP is PSpace-complete, whereas that of MDP is $\mathrm{P}$ complete [10]
} 
in terms of $\alpha$-vectors ${ }^{2}$ over a finite number of points in the reachable set [16]. Note that unlike grid-based approaches, here the points are not fixed and may change as the value function changes. In policy-search methods such as finite-state controllers, attention is restricted to a certain class (structure) of strategies, and the objective is to find the best strategy in that class using policy iteration and gradient-based techniques [17]. The above methods often use the belief space as the planning space, whose size grows exponentially with the number of nodes and time horizon; thus, they suffer from the curse of dimensionality. In addition, the dynamics of the belief state depends on the transition probability matrix (i.e., it is a modelbased state). Hence, it is not clear how the above POMDP solvers may be used in the case where the model is not exactly known [18]. For more details on POMDPs, the interested reader is referred to [18, 19] and references therein.

In this paper, it is desired to find a near-optimal strategy that determines, at each time instant, whether to collect the data or to estimate them. In contrast to POMDP solvers mentioned in the previous paragraph, we exploit the structure of the problem to efficiently solve the resultant POMDP by using a different planning space (which is smaller than the belief space and is independent of the model) 3 To determine the complexity of the above problem, it is noted that the information structure is imperfect, and the model structure is not necessarily complete (known), yet the decision maker wishes to sequentially choose the frequency of collecting data based on several factors. Some of such factors include, for example, how informative the data are at each time instant to be fed to the estimator, or, how costly and credible that data are upon collection, and how quickly the states of nodes change with time. Three examples are provided to illustrate the efficacy of the proposed strategy. The first example studies a sensor network, where a sensor measures a dynamic phenomenon with saturation levels such as the temperature of a room, and reports it to a data center over an unreliable link at some communication cost in such a way that the estimate constructed at the data center is reliable. The second example studies a communication network with two different topologies, where the weighted average of an arbitrary number of Markov processes with linear dynamics is to be collected at some transmission cost. The third example deals with a social network, where an agency is to conduct a survey from a number of users by offering monetary rewards as an incentive for participation.

The paper is organized as follows. In Section II the problem is formulated for both known and unknown models. To facilitate the analysis, some preliminary results on the dynamics of the empirical distribution are derived in Section III. The proposed solutions are then presented in Section IV followed by the asymptotic analysis in Section $\mathrm{V}$. The special case of linear dynamics is studied in Section VI The extension of

\footnotetext{
${ }^{2}$ The notion of $\alpha$-vectors was introduced in [13] to solve the finite-horizon POMDP, and was later enhanced by pruning dominated vectors [14 15].

${ }^{3}$ The idea of defining a pseudo-state to plan and learn in POMDPs by taking the structure of the problem into account was first introduced in 20 Chapters 5,6] and a preliminary result was presented in [21]. In particular, the pseudo-state (called incrementally expansion representation) provides a guaranteed optimality bound, and is more general than (model-based) belief state and (model-free) history state.
}

the obtained results to more complex networks is discussed in Section VII. To demonstrate the efficacy of the proposed strategies, three numerical examples are provided in Section VIII Finally, some concluding remarks are given in Section IX

\section{A. Notation}

Throughout this paper, $\mathbb{N}, \mathbb{Z}$ and $\mathbb{R}$ denote the set of natural, integer and real numbers, respectively. For any $k \in$ $\mathbb{N}$, the shorthand notations $\mathbb{N}_{k}, \mathbb{N}_{k}^{*}$ and $\mathbb{Z}_{k}$ are used to represent the finite set of integers $\{1, \ldots, k\},\{0,1, \ldots, k\}$ and $\{-k, \ldots, 0, \ldots, k\}$, respectively. Given vectors $a, b, c$, $\operatorname{vec}(a, b, c)=\left[a^{\top}, b^{\top}, c^{\top}\right]^{\top}$. For any $a, b \in \mathbb{N}, a \leq b, x_{a: b}$ is defined as the vector $\left(x_{a}, x_{a+1}, \ldots, x_{b}\right)$. Moreover, $\mathbb{1}(\cdot)$ is the indicator function, $\mathbb{P}(\cdot)$ is the probability of an event, $\mathbb{E}[\cdot]$ is the expectation of a random variable, $\|\cdot\|$ is the infinity norm of a vector, $\operatorname{Tr}(\cdot)$ is the trace of a matrix, and $\operatorname{diag}(\cdot)$ is a diagonal matrix. Given a finite set $\mathcal{S}, \mathcal{P}(\mathcal{S})$ denotes the space of probability measures over set $\mathcal{S}$, and $|\mathcal{S}|$ is the size of this finite set. The notation $\operatorname{binopdf}(n, p)$ is used for the binomial probability distribution function with $n \in \mathbb{N}$ trials, and success probability $p \in[0,1]$. For any square matrix $A$, $A^{0}$ is the identity matrix of the same size as $A$.

\section{B. Main contributions}

It is generally difficult to find the optimal control strategy when the information structure is imperfect because of the dual effect phenomenon [22], where the control strategy affects the estimation process by altering the observation dynamics while the estimation strategy impacts the control strategy by determining the state estimate. As a result, it is of particular interest in control theory to identify decision-making problems with imperfect information structure that admit tractable solutions. The main contributions of this paper are outlined below.

1) A theoretical analysis is provided for an important trade-off that emerges in different forms in networked systems, namely, collecting data versus estimating data. In particular, we exploit the structure of the problem to introduce a new planning space, which is different from the conventional belief space (Theorem 3 . The salient feature of the proposed space is that it leads to a low-complexity near-optimal solution when the model is known (Theorem 47). Furthermore, the solution methodology can be extended to the case of systems with unknown models while such an extension in the belief space is conceptually difficult because the belief space is model-dependent. More precisely, we develop reinforcement learning algorithms to deal with unknown probability distributions and unmodelled dynamics (Proposition 2 and Theorem 5).

2) To provide a more practical setup, we define a Bernoulli probability distribution to design a robust strategy in order to embody data uncertainty. We show that our results naturally extend to the case where observations are received with a constant time delay. We also study the role of the number of nodes, and define a certainty threshold that determines when data estimation becomes 
more desirable than data collection, as the number of nodes increases (Theorem 6 and Corollary 3 .

3) For the special case of linear dynamic systems, we establish a separation principle between control and estimation (that also holds for non-Gaussian random variables), which is different from the existing result for only Gaussian random variables (Theorem 7). We then provide an efficient dynamic-program-based solution for computing a near-optimal scheduling strategy, where the proposed planning space requires no knowledge of the underlying probability distributions and corresponding matrices (Theorem 8). It is to be noted that our proposed strategy is nonlinear even in the case of linear dynamics with quadratic cost function.

4) We show that the computational complexity of the proposed strategy is linear with respect to the approximation index, to be defined later, and polynomial with respect to the number of nodes. For the special case of linear quadratic cost functions, the complexity of computing the strategy is independent of the number of nodes as well as the dimension of the state space.

5) We generalize the obtained results to the following cases: (a) multiple decision makers and multiple estimators; (b) multiple reset actions; (c) partially exchangeable and partially equivariant networks, and (d) Markovian noise and Markovian credibility processes (see Section VII for details).

\section{Problem Formulation}

Consider an index-invariant (exchangeable) network of $n \in$ $\mathbb{N}$ nodes. Let $s_{t}^{i} \in \mathcal{S}$ denote the state of node $i \in \mathbb{N}_{n}$ at time $t \in \mathbb{N}$, where $\mathcal{S} \subset \mathbb{R}$ is a known finite set. Denote by $m_{t} \in \mathcal{M}(n)$ the empirical distribution of states at time $t$, i.e.,

$$
m_{t}(s)=\frac{1}{n} \sum_{i=1}^{n} \mathbb{1}\left(s_{t}^{i}=s\right), \quad s \in \mathcal{S},
$$

where $\mathcal{M}(n)=\left\{\left(\alpha_{1}, \ldots, \alpha_{|\mathcal{S}|}\right) \mid \alpha_{i} \in\left\{0, \frac{1}{n}, \ldots, 1\right\}, i \in\right.$ $\left.\mathbb{N}_{|\mathcal{S}|}, \sum_{i=1}^{|\mathcal{S}|} \alpha_{i}=1\right\}$ is the set of empirical distributions over the state space $\mathcal{S}$ with $n$ samples. In the sequel, the empirical distribution of states is sometimes referred to as data. It is shown in [20] that any set of exchangeable Markov processes $\mathbf{s}_{t}=\operatorname{vec}\left(s_{t}^{1}, \ldots, s_{t}^{n}\right)$ can be equivalently expressed as a set of Markov processes coupled through the empirical distribution of states. Therefore, let the state of the $i$-th node at time $t$ evolve according to the following dynamics:

$$
s_{t+1}^{i}=f\left(s_{t}^{i}, m_{t}, w_{t}^{i}\right), \quad i \in \mathbb{N}_{n}, t \in \mathbb{N},
$$

where $w_{t}^{i} \in \mathcal{W} \subset \mathbb{R}$ is the local noise of node $i$ at time $t$. In addition, consider a decision maker that wishes to find an affordable way to sample the data over time horizon such that the estimate of the data, constructed based on the previously sampled data, is reliable. Let $a_{t} \in \mathcal{A}:=\{0,1\}$ denote the action of the decision maker at time $t \in \mathbb{N}$, where $a_{t}=1$ means that the decision maker collects the data and $a_{t}=0$ means that it does not collect them.

In real-world applications, it is possible that the collected data are not credible due to, for instance, misinformation induced by fake news in social networks, packet drop in communication networks, and faulty encoders and decoders in sensor networks. Denote by $q \in[0,1]$ the probability that the collected data are credible (correct). When the data are not credible, they are discarded. Note that the evaluation of the credibility of the data may be viewed as an exogenous process that can have any arbitrary Markov-chain dynamics. This extension does not add much complexity to our analysis, and is not considered here for simplicity of notation.

Denote by $o_{t} \in \mathcal{O}:=\mathcal{M}(n) \cup\{$ blank $\}$ the observation of the decision maker at time $t$, where blank implies that the decision maker receives either no observation, when $a_{t}=0$, or potentially incorrect (unreliable) data, when $a_{t}=1$. Subsequently, for any $t \in \mathbb{N}$ and $m \in \mathcal{M}(n)$,

$$
\mathbb{P}\left(o_{t+1}=\mathrm{blank} \mid m_{t+1}=m, a_{t}=0\right)=1,
$$

and

$$
\begin{gathered}
\mathbb{P}\left(o_{t+1}=m \mid m_{t+1}=m, a_{t}=1\right)=q, \\
\mathbb{P}\left(o_{t+1}=\text { blank } \mid m_{t+1}=m, a_{t}=1\right)=1-q,
\end{gathered}
$$

where initially $o_{1}=m_{1}$. The decision maker determines its action at time $t$ based on its information by that time, i.e.,

$$
a_{t}=g_{t}\left(o_{1: t}, a_{1: t-1}\right),
$$

where $g_{t}: \mathcal{O}^{t} \times \mathcal{A}^{t-1} \rightarrow \mathcal{A}$ is called the control law of the decision maker. Denote by $g:=\left\{g_{1}, g_{2}, \ldots\right\}$ the strategy of the decision maker. Note that the information structure of the decision maker is imperfect because it only has access to the collected data.

Let $\mathbf{w}_{t}=\operatorname{vec}\left(w_{t}^{1}, \ldots, w_{t}^{n}\right) \in \mathcal{W}^{n}, t \in \mathbb{N}$, with a probability distribution function $P_{\mathbf{w}}$. Let also $\eta_{t} \in\{0,1\}$ denote the random variable representing the credibility process at time $t$ such that $\mathbb{P}\left(\eta_{t}=1\right)=q$. It is assumed that the primitive random variables $\left\{\mathbf{s}_{1}, \mathbf{w}_{1}, \mathbf{w}_{2}, \ldots, \eta_{1}, \eta_{2}, \ldots\right\}$ are defined on a common probability space, are mutually independent, and have finite variances. At any time $t \in \mathbb{N}$, the decision maker constructs an estimate of the data $m_{t}$, denoted by $\hat{m}_{t} \in \mathcal{M}(n)$, according to an estimator function $h$ as follows:

$$
\hat{m}_{t}=h\left(\mathbb{P}\left(m_{t} \mid o_{1: t}, a_{1: t-1}\right)\right) .
$$

The objective of the decision maker is to design a strategy that not only keeps the estimation error small but also incurs minimal collection cost. To this end, we define the following per-step cost $c: \mathcal{M}(n)^{2} \times \mathcal{A} \rightarrow \mathbb{R}_{\geq 0}$, i.e.

$$
c\left(m_{t}, \hat{m}_{t}, a_{t}\right)
$$

where $c\left(m_{t}, \hat{m}_{t}, 0\right)$ is the cost when the decision maker chooses not to collect data at time $t$, and $c\left(m_{t}, \hat{m}_{t}, 1\right)$ is the cost when it is decided to collect data.

\section{A. Linear dynamics}

Since the role of topology is implicitly described in (2), we present an equivariant linear network in this subsection whose topology can be described explicitly in terms of the eigenvalues and eigenvectors of its adjacency matrix. In particular, consider an undirected weighted graph with a real-valued 
symmetric adjacency matrix A. One standard way to vectorize the graph is to use the spectral decomposition [23], i.e.,

$$
\mathbf{A} \approx\left[\sum_{l=0}^{L} \alpha(l)(\mathbf{A})^{l}\right]=\left[\sum_{l=0}^{L} \alpha(l)\left(\sum_{j=1}^{n} \lambda_{j} \mathbf{V}(:, j) \mathbf{V}(:, j)^{\boldsymbol{\top}}\right)^{l}\right],
$$

where $\alpha(l) \in \mathbb{R}, \mathbf{V}$ is an orthogonal matrix consisting of the eigenvectors of $\mathbf{A}, \Lambda$ is a diagonal matrix of the corresponding eigenvalues, and $\mathbf{A}^{l}=\mathbf{V} \Lambda^{l} \mathbf{V}^{\top}=\sum_{j=1}^{n} \lambda_{j}^{l} \mathbf{V}(:, j) \mathbf{V}(:, j)^{\top}$, $l \in \mathbb{N}_{L}$. Let $D \ll n$ be the number of "dominant" eigenvalues. Then, the dynamics of the network can be expressed as follows:

$$
\begin{aligned}
\mathbf{s}_{t+1} & =\mathbf{A} \mathbf{s}_{t} \approx\left[\sum_{l=0}^{L} \alpha(l)\left(\sum_{d=1}^{D} \lambda_{d} \mathbf{V}(:, d) \mathbf{V}(:, d)^{\boldsymbol{\top}}\right)^{l}\right] \mathbf{s}_{t} \\
& =\sum_{d=1}^{D} A_{d} \mathbf{V}(:, d) m_{t}^{d},
\end{aligned}
$$

where $m_{t}^{d}:=\mathbf{V}(:, d)^{\top} \mathbf{s}_{t}=\sum_{i=1}^{n} \mathbf{V}(i, d) s_{t}^{i}$ and $A_{d}:=$ $\sum_{l=0}^{L} \alpha(l)\left(\lambda_{d}\right)^{l}, d \in \mathbb{N}_{D}$. Hence, for any mode $d \in \mathbb{N}_{D}$ :

$$
m_{t+1}^{d}=\mathbf{V}(:, d)^{\top} \mathbf{s}_{t+1} \approx A_{d} m_{t}^{d},
$$

where $\mathbf{V}(:, d)^{\top} \mathbf{V}(:, j)$ is equal to the Kronecker delta function $\delta_{d, j}$ for any $d, j \in \mathbb{N}_{n}$. Based on the above vectorized representation, consider now a network of $n \in \mathbb{N}$ nodes, wherein the state of node $i \in \mathbb{N}_{n}$ at time $t \in \mathbb{N}$ is a vector denoted by $s_{t}^{i} \in \mathbb{R}^{d_{s}}$. Let $m_{t}^{d}$ be the weighted average of the states at time $t$ associated with the $d$-th dominant mode, i.e.

$$
m_{t}^{d}:=\frac{1}{n} \sum_{i=1}^{n} v^{i, d} s_{t}^{i}, \quad \bar{w}_{t}^{d}=\frac{1}{n} \sum_{i=1}^{n} v^{i, d} w_{t}^{i},
$$

where $d$-th eigenvector is normalized as $\frac{1}{n} \sum_{i=1}^{n}\left(v^{i, d}\right)^{2}=1$, and $w_{1: \infty}^{i}$ is a random process with zero mean and finite covariance matrix with a known bound $\Sigma^{i, w} \leq \Sigma_{\max } \in \mathbb{R}^{d_{s} \times d_{s}}$. Let the dynamics of the augmented weighted average be described by the following linear equation:

$$
m_{t+1}=A m_{t}+\bar{w}_{t}
$$

where $m_{t}=\operatorname{vec}\left(m_{t}^{1}, \ldots, m_{t}^{D}\right), A=\operatorname{diag}\left(A_{1}, \ldots, A_{D}\right)$, and $\bar{w}_{t}=\operatorname{vec}\left(\bar{w}_{t}^{1}, \ldots, \bar{w}_{t}^{D}\right)$. The per-step cost function at time $t \in \mathbb{N}$ is defined as:

$$
c\left(m_{t}, \hat{m}_{t}, a_{t}\right):=\left(m_{t}-\hat{m}_{t}\right)^{\top}\left(m_{t}-\hat{m}_{t}\right) z\left(a_{t}\right)+\ell\left(m_{t}, a_{t}\right),
$$

where $\hat{m}_{t}:=h_{t}\left(o_{1: t}, a_{1: t-1}\right) \in \mathbb{R}^{D d_{s}}$ is a generic nonlinear estimator and $z, \ell$ are real-valued non-negative functions, i.e., $z: \mathcal{A} \rightarrow \mathbb{R}_{\geq 0}$ and $\ell: \mathbb{R}^{D d_{s}} \times \mathcal{A} \rightarrow \mathbb{R}_{\geq 0}$.

\section{B. Problem statement}

Let $J(g)$ be the total expected discounted cost given by:

$$
J(g)=\mathbb{E}^{g}\left[\sum_{t=1}^{\infty} \gamma^{t-1} c\left(m_{t}, \hat{m}_{t}, a_{t}\right)\right],
$$

where $\gamma \in(0,1)$ is the discount factor (which is an incentive parameter to push for a decision early rather than postponing it indefinitely) and the expectation in (11) is taken with respect to the probability measures induced on the sample paths by the choice of strategy $g$.

Problem 1. Given $\varepsilon \in \mathbb{R}_{>0}$, it is desirable to develop an $\varepsilon$-optimal strategy $g_{\varepsilon}^{*}$ such that for any strategy $g$,

$$
J\left(g_{\varepsilon}^{*}\right) \leq J(g)+\varepsilon .
$$

Problem 2. When the knowledge of the model $\left(f, q, c, P_{\mathrm{w}}\right)$ is incomplete, it is desirable to find a reinforcement learning $(R L)$ algorithm that converges to an $\varepsilon$-optimal strategy $g_{\varepsilon}^{*}$ satisfying inequality (12).

In what follows, we first study the Markov-chain model and then investigate the special case of linear dynamics.

\section{DYNAMICS OF DATA}

Prior to addressing Problems 1 and 2, it is necessary to analyze the evolution of data in time. Many natural systems obey some form of the invariance principle. For example, the outcome of an election is independent of voters' identity (index of the voters), the spectrum of the adjacency matrix of an undirected graph does not depend on the specific labeling of the nodes, and the power demand of a user in a smart grid is often independent of other users' demands. Hence, it is reasonable to assume that the local noises are exchangeable (index-invariant) and i.i.d. (independent and identically distributed). It is to be noted that these are standard assumptions in statistical models and data science [24].

Assumption 1. The primitive random variables $w_{t}^{1}, \ldots, w_{t}^{n}$ are exchangeable at any time $t \in \mathbb{N}$.

Proposition 1. Let Assumption 1 hold. The empirical distribution $m_{t}, t \in \mathbb{N}$, is a Markov process and evolves almost surely at any state $s \in \mathcal{S}$ as follows:

$$
m_{t+1}\left(s^{\prime}\right)=\sum_{s \in \mathcal{S}} \sum_{w \in \mathcal{W}} m_{t}(s) \mathbb{1}\left(f\left(s, m_{t}, w\right)=s^{\prime}\right)\left[\frac{1}{n} \sum_{j=1}^{n} \mathbb{1}\left(w_{t}^{i}=w\right)\right] \text {. }
$$

Proof. The proof follows directly from the fact that the empirical distribution of states and also the primitive random variables are invariant to the permutation of nodes.

Assumption 2. The primitive random variables $w_{t}^{1}, \ldots, w_{t}^{n}$ are i.i.d. at any time $t \in \mathbb{N}$ with probability function $P_{W}$.

Under Assumption 2, the dynamic equation (2) can also be described in terms of the transition probability matrix at any $s^{\prime}, s \in \mathcal{S}$ and $m \in \mathcal{M}(n)$ as follows:

$$
\begin{aligned}
T\left(s^{\prime}, s, m\right) & :=\mathbb{P}\left(s_{t+1}^{i}=s^{\prime} \mid s_{t}^{i}=s, m_{t}=m\right) \\
& =\sum_{w \in \mathcal{W}} P_{W}\left(w_{t}^{i}=w\right) \mathbb{1}\left(s^{\prime}=f(s, m, w)\right),
\end{aligned}
$$

where the probability of transitioning to state $s^{\prime}$ from state $s$, given the empirical distribution $m$, is equal to the probability of realizations $w$ resulting in this transition. For any $s^{\prime}, s \in \mathcal{S}$ and $m \in \mathcal{M}(n)$, define the vector-valued function $\phi_{m(s)}$ : $\mathcal{S}^{2} \times \mathcal{M}(n) \rightarrow \mathcal{P}(\{0,1, \ldots, n \cdot m(s)\})$ as follows:

$$
\begin{aligned}
& \phi_{m(s)}\left(s^{\prime}, s, m\right)=\delta_{0}(n \cdot m(s)) \\
&+\mathbb{1}(m(s)>0) \operatorname{binopdf}\left(n \cdot m(s), T\left(s^{\prime}, s, m\right)\right),
\end{aligned}
$$


where $\delta_{0}(n \cdot m(s))$ is a Dirac measure with the domain set $\{0,1, \ldots, n \cdot m(s)\}$ and a unit mass concentrated at zero. In addition, let $\bar{\phi}: \mathcal{S} \times \mathcal{M}(n) \rightarrow \mathcal{P}(\{0,1, \ldots, n\})$ be the convolution of $\phi_{m(s)}\left(s^{\prime}, s, m\right)$ over all states $s \in \mathcal{S}=$ $\left\{s_{1}, \ldots, s_{|\mathcal{S}|}\right\}$, i.e.,

$$
\bar{\phi}\left(s^{\prime}, m\right)=\phi_{m\left(s_{1}\right)}\left(s^{\prime}, s_{1}, m\right) * \ldots * \phi_{m\left(s_{|\mathcal{S}|}\right)}\left(s^{\prime}, s_{|\mathcal{S}|}, m\right),
$$

where $\bar{\phi}\left(s^{\prime}, m\right)$ is a vector of size $n+1$. When the primitive random variables are independent and identically distributed, the evolution of data has a special structure as described in the next theorem.

Theorem 1 (Deep Chapman-Kolmogorov equation [3]). Let Assumption 2 hold. Given $m_{t} \in \mathcal{M}(n)$ at time $t \in \mathbb{N}$, the transition probability matrix of the empirical distribution can be obtained as follows:

$$
\mathbb{P}\left(m_{t+1}\left(s^{\prime}\right)=\frac{y}{n} \mid m_{t}\right)=\bar{\phi}\left(s^{\prime}, m_{t}\right)(y+1), s^{\prime} \in \mathcal{S}, y \in \mathbb{N}_{n}^{*} .
$$

Proof. The proof is presented in Appendix A

To simplify the notation, denote by $T_{\mathrm{m}}\left(m^{\prime}, m\right)$ the transition probability matrix of the empirical distribution described in Proposition 1 and Theorem 1 , i.e.,

$$
T_{\mathrm{m}}\left(m^{\prime}, m\right):=\mathbb{P}\left(m_{t+1}=m^{\prime} \mid m_{t}=m\right), \quad m^{\prime}, m \in \mathcal{M}(n) .
$$

In general, the complexity of computing $T_{\mathrm{m}}$ in time is exponential with respect to the number of nodes $n$. However, when the local noises are exchangeable, this complexity reduces to polynomial time according to Proposition 1 (because the space of empirical distributions grows polynomially with respect to $n[20]$ ). The above complexity can be further alleviated in time when the noises are i.i.d., according to Theorem 1 .

Theorem 2. Suppose Assumption 2 holds and the dynamics of the states in (2) are decoupled, i.e., $s_{t+1}^{i}=f\left(s_{t}^{i}, w_{t}^{i}\right)$, $i \in \mathbb{N}_{n}, t \in \mathbb{N}$. The transition probability matrix $T_{\mathrm{m}}\left(m^{\prime}, m\right)$, whose size $|\mathcal{M}(n)|^{2}$ increases with $n$, can be identified by the transition probability matrix $T\left(s^{\prime}, s\right)$, whose size $|\mathcal{S}|^{2}$ is independent of $n$.

Proof. The proof follows directly from (13) and Theorem 1 on noting that $T\left(s^{\prime}, s, m\right)$ reduces to $T\left(s^{\prime}, s\right)$ for decoupled dynamics. In this case, knowing function $T\left(s^{\prime}, s\right), s^{\prime}, s \in \mathcal{S}$, is enough to compute the function $\phi_{m(s)}, m \in \mathcal{M}(n)$, and subsequently function $\bar{\phi}$ in (14). Therefore, one can determine the global interactions $T_{\mathrm{m}}\left(m^{\prime}, m\right), m^{\prime}, m \in \mathcal{M}(n)$ by identifying the local interactions $T\left(s^{\prime}, s\right), s^{\prime}, s \in \mathcal{S}$.

Remark 1. A consequence of Theorem 2 is that the transition probability matrix of data $T_{\mathrm{m}}$ can be identified by $|\mathcal{S}|^{2}$ scalars, which is a considerable reduction in the parameter space.

\section{Near-Optimal strategies for Problems 1 and 2}

In this section, we propose near-optimal strategies for Problems 1 and 2. At any time $t \in \mathbb{N}$, let $x_{t} \in \mathcal{M}(n)$ denote the last credible data (i.e., the last observation of the decision maker that is not blank). Let also $y_{t} \in \mathbb{N}^{*}$ be the number of blanks up to time $t$, after the last credible data $x_{t}$. The initial value of $\left(x_{t}, y_{t}\right)$ is $\left(m_{1}, 0\right)$ because $o_{1}=m_{1}$. When data are credible upon request (i.e. $q=1$ ), the number of blanks $y$ has an inverse relationship with the frequency of collecting data that is $1 /(y+1)$.

In the following lemma, we identify the dynamics of $\left(x_{t+1}, y_{t+1}\right)$ at time $t \in \mathbb{N}$, given $\left(x_{t}, y_{t}, o_{t+1}\right)$.

Lemma 1. There exists a function $\hat{f}: \mathcal{M}(n) \times \mathbb{N}^{*} \times \mathcal{O} \rightarrow$ $\mathcal{M}(n) \times \mathbb{N}^{*}$ such that $\left(x_{t+1}, y_{t+1}\right)=\hat{f}\left(x_{t}, y_{t}, o_{t+1}\right), t \in \mathbb{N}$, i.e.,

$$
\hat{f}\left(x_{t}, y_{t}, o_{t+1}\right):= \begin{cases}\left(x_{t}, y_{t}+1\right), & o_{t+1}=\mathrm{blank}, \\ \left(o_{t+1}, 0\right), & o_{t+1} \neq \mathrm{blank} .\end{cases}
$$

Proof. The proof follows from the definition of $x_{t}$ and $y_{t}$.

Now, let the observation $o_{t} \in \mathcal{O}, t \in \mathbb{N}$, be rewritten as:

$$
o_{t}=x_{t} \text {, if } y_{t}=0, \quad \text { and } o_{t}=\mathrm{blank} \text {, if } y_{t} \neq 0 \text {. }
$$

According to (16) and (17), one can conclude that sets $\left\{o_{1: t}, a_{1: t-1}\right\}$ and $\left\{x_{1: t}, y_{1: t}, a_{1: t-1}\right\}$ have equivalent information as each set can be fully specified by the other one, i.e.,

$\mathbb{P}\left(m_{t} \mid o_{1: t}, a_{1: t-1}\right)=\mathbb{P}\left(m_{t} \mid x_{1: t}, y_{1: t}, a_{1: t-1}\right)=T_{\mathrm{m}}^{y_{t}}\left(m_{t}, x_{t}\right)$,

where $T_{\mathrm{m}}^{y_{t}}$ is the transition matrix (15) to the power of $y_{t}$.

In the next lemma, we demonstrate that $\left(x_{t+1}, y_{t+1}\right)$ has Markovian dynamics by showing that the posterior probability of $o_{t+1}$ given the history $\left(x_{1: t}, y_{1: t}, a_{1: t}\right)$ depends only on the information at time $t$, i.e., $\left(x_{t}, y_{t}, a_{t}\right)$.

Lemma 2. Let Assumption 1 hold. Given any realization $x_{1: t}, y_{1: t}$ and $a_{1: t}, t \in \mathbb{N}$, the following equality holds irrespective of the strategy $g$ :

$$
\begin{gathered}
\mathbb{P}\left(o_{t+1} \mid x_{1: t}, y_{1: t}, a_{1: t}\right)=\left(1-a_{t} q\right) \cdot \mathbb{1}\left(o_{t+1}=\mathrm{blank}\right) \\
+a_{t} \cdot q \cdot T_{\mathrm{m}}^{y_{t}+1}\left(o_{t+1}, x_{t}\right) \cdot \mathbb{1}\left(o_{t+1} \neq \mathrm{blank}\right) .
\end{gathered}
$$

Proof. The proof is presented in Appendix B

We now prove that the conditional expectation of the perstep cost given the history of information by time $t$ can be described by the information at time $t$.

Lemma 3. Given any realization $x_{1: t}, y_{1: t}$ and $a_{1: t}, t \in \mathbb{N}$, there exists a function $\hat{c}: \mathcal{M}(n) \times \mathbb{N}^{*} \times \mathcal{A} \rightarrow \mathbb{R}_{\geq 0}$ such that:

$$
\begin{aligned}
\mathbb{E}\left[c\left(m_{t}, \hat{m}_{t}, a_{t}\right) \mid x_{1: t}, y_{1: t}, a_{1: t}\right]=\hat{c}\left(x_{t}, y_{t}, a_{t}\right) \\
:=\sum_{m \in \mathcal{M}(n)} c\left(m, h\left(T_{\mathrm{m}}^{y_{t}}\left(m, x_{t}\right)\right), a_{t}\right) T_{\mathrm{m}}^{y_{t}}\left(m, x_{t}\right),
\end{aligned}
$$

where the above equality holds irrespective of strategy $g$.

Proof. The proof is presented in Appendix C.

From the results of Lemmas 1,3 an optimal strategy for Problem 11 is identified by the following theorem.

Theorem 3. Let Assumption 1 hold. The optimal solution of Problem 1 is given by the following Bellman equation such that for any $x \in \mathcal{M}(n)$ and $y \in \mathbb{N}^{*}$,

$$
V(x, y)=\min _{a \in \mathcal{A}}(\hat{c}(x, y, a)+\gamma \mathbb{E}[V(\hat{f}(x, y, o))]),
$$


where the above expectation is taken over all observations $o \in \mathcal{O}$ with probability distribution (19).

Proof. The proof follows from the fact that $\left(x_{t}, y_{t}\right)$ are sufficient statistic to identify the optimal solution of Problem 1 More precisely, given observations $o_{1: t}, t \in \mathbb{N}$, state $\left(x_{t}, y_{t}\right)$ is observable at any time $t$ and has Markovian dynamics according to Lemma 1. In addition, given any history $\left(x_{1: t}, y_{1: t}, a_{1: t}\right)$, the conditional probability of $o_{t+1}$ and the conditional expectation of the per-step cost $c\left(m_{t}, \hat{m}, a_{t}\right)$ do not depend on the strategy $g$ according to Lemmas 2 and 3 . respectively. Consequently, the optimal solution of Problem 1 can be identified by dynamic programming decomposition, and Bellman equation (21) is obtained from well-known results in Markov decision theory [25, Proposition 5.4.1, Volume 1].

Since the planning space in Theorem 3 is countably infinite, the solution of the Bellman equation (21) is intractable, in general. As a result, we propose an $\varepsilon$-optimal solution based on a truncation technique whose performance is within an arbitrary neighbourhood (determined by $\varepsilon$ ) of the optimal performance for Problem 1 .

Remark 2. Under some practical constraints such as limited energy resources for data collection or saturation of states, the feasible set of the dynamic program (21) may reduce to a finite set, yielding a tractable optimization problem.

\section{A. An E-optimal solution for Problem 1}

Denote by $c_{\max }$ an upper bound on the per-step cost (7). For any $k \in \mathbb{N}$, define the following Bellman equation at any $x, m^{*} \in \mathcal{M}(n)$ and $y \in \mathbb{N}_{k}^{*}$ :

$$
\tilde{V}_{k}(x, y)=\min \left(\tilde{V}_{k}^{0}(x, y), \tilde{V}_{k}^{1}(x, y)\right),
$$

where

$$
\left\{\begin{array}{c}
\tilde{V}_{k}^{0}(x, y):=\sum_{m \in \mathcal{M}(n)} c\left(m, h\left(T_{\mathrm{m}}^{y}(m, x)\right), 0\right) T_{\mathrm{m}}^{y}(m, x) \\
+\gamma\left(\mathbb{1}(y<k) \tilde{V}_{k}(x, y+1)+\mathbb{1}(y=k) \tilde{V}_{k}\left(m^{*}, 0\right)\right), \\
\tilde{V}_{k}^{1}(x, y):=\sum_{m \in \mathcal{M}(n)} c\left(m, h\left(T_{\mathrm{m}}^{y}(m, x)\right), 1\right) T_{\mathrm{m}}^{y}(m, x) \\
+(1-q) \gamma\left(\mathbb{1}(y<k) \tilde{V}_{k}(x, y+1)+\mathbb{1}(y=k) \tilde{V}_{k}\left(m^{*}, 0\right)\right) \\
\quad+q \gamma\left(\sum_{m^{\prime} \in \mathcal{M}(n)} T_{\mathrm{m}}^{y+1}\left(m^{\prime}, x\right) \tilde{V}_{k}\left(m^{\prime}, 0\right)\right) .
\end{array}\right.
$$

Theorem 4. Suppose Assumption 1 holds. Given any $\varepsilon \in \mathbb{R}_{>0}$, let $k(\varepsilon) \in \mathbb{N}$ be sufficiently large such that

$$
k(\varepsilon) \geq \log \left(\frac{(1-\gamma) \varepsilon}{2 c_{\max }}\right) / \log (\gamma) .
$$

Then, using $k=k(\varepsilon)$, any solution to the Bellman equation (22) is an E-optimal solution for Problem 1, i.e.,

$$
g_{\varepsilon}^{*}(x, y):= \begin{cases}0, & \tilde{V}_{k}^{0}(x, y) \leq \tilde{V}_{k}^{1}(x, y), \\ 1, & \tilde{V}_{k}^{0}(x, y)>\tilde{V}_{k}^{1}(x, y) .\end{cases}
$$

Proof. The proof is presented in Appendix D

To numerically compute the solution of the Bellman equation (22), one can use value iteration, policy iteration, or any other existing approximate method [25, 26]. Note that the space of Bellman equation (22) (i.e., $\overline{\mathcal{M}}(n) \times \mathbb{N}_{k}^{*}$ ) grows polynomially with the number of nodes $n$ and linearly with the approximation index $k$. For the case when (23) is equality, $k(\varepsilon)$ is proportional to $-\log ($ constant $\times \varepsilon)$. Note that $\gamma \in(0,1)$, which means its $\log$ is negative. Hence, $-\log (\operatorname{constant} \times \varepsilon)=$ $\log \left(\right.$ constant $\left.\times \varepsilon^{-1}\right)$. Therefore, the following result holds.

Corollary 1. The computational complexity of the proposed solution in Theorem 4 is linear with respect to the approximation index $k(\varepsilon)$, logarithmic with respect to the inverse of the size of the desired neighborhood $\varepsilon$, and polynomial with respect to the number of nodes $n$.

Corollary 2. For the special case of single node, i.e., $n=1$, there is no loss of optimality in replacing space $\mathcal{M}(n)$ by space $\mathcal{S}$ in Theorems 3 and 4

Proof. The proof follows on noting that when $n=1$, spaces $\mathcal{M}(n)$ and $\mathcal{S}$ have equivalent information, i.e., $m_{t}=\delta_{s_{t}}$ at any time $t \in \mathbb{N}$.

Remark 3. It is to be noted that the application domain of the present work is different from applications such as sensor selection, where the objective is to dynamically choose a subset of sensors in order to monitor a time-varying phenomenon [27 28]. For example, in sleep sensor scheduling control [28] the dynamics of the target (phenomenon) is decoupled from the scheduling (sampling) strategy, the value of data at each time instant is binary (i.e., it is zero if data is observed and it is one otherwise), and the planning space consists of the belief state of the target as well as the residual sleep times of sensors [28. Theorem 3.1]. In contrast, the phenomenon considered in this paper is an estimate of data, generated by the estimator, that is influenced by the sampling strategy, and leads to a dual effect [22]. The value of data depends on various parameters such as the cost and estimator functions and is not necessarily a binary variable. Furthermore, the proposed dynamic program is based on a planning space, defined as the last credible data and the elapsed time since then (which is not a belief space). In addition, the action set here does not depend on the number of nodes and the state space and the dynamics of the phenomenon (data), and the solution methodology is amenable to the incompleteness of the model structure.

\section{B. An E-optimal solution for Problem 2}

Two different approaches are considered here to find a nearoptimal strategy for Problem 2. The first one is an indirect (model-based) approach which involves two steps: supervised learning and planning. Given a large number of training samples, one can utilize supervised-learning (parametrization) techniques such as linear regression and logistic regression to identify the model, and then find the planning solution of the Bellman equation 22 by using methods such as value iteration and policy iteration [25]. In general, the total number of unknown parameters that should be learned to solve equation (22) using this approach is equal to $|\mathcal{M}(n)|^{2}+2(k+$ 1) $|\mathcal{M}(n)|+1$, with $|\mathcal{M}(n)|^{2}$ scalars for transition probability matrix $T_{\mathrm{m}},\left|\mathcal{M}(n)\left\|\mathbb{N}_{k}^{*}\right\| \mathcal{A}\right|$ scalars for the per-step cost $c$, and 1 scalar for the credibility of data $q$. In practice, the first approach is feasible when the number of unknown parameters is relatively small. For example, the deep Chapman-Kolmogorov 


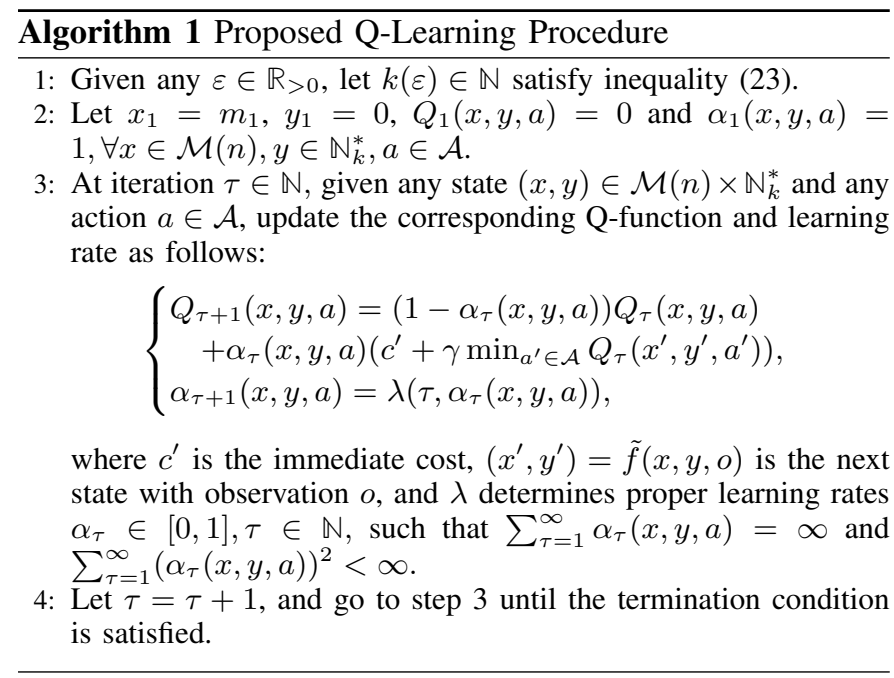

equation in Theorem 1 can be parametrized by a small number of variables according to Theorem 2, for the case when the dynamics of nodes are decoupled and the random variables are i.i.d., which is a non-trivial (yet efficient) parametrization.

Proposition 2. Let Assumption 2 hold and the per-step cost function as well as the estimator function be given. When the dynamics of the states are decoupled and $n$ is large, it is more efficient to first learn the model and then solve the Bellman equation (22) to obtain an $\varepsilon$-optimal solution for Problem 2

Proof. The proof follows on noting that the total number of unknown parameters in 22 is $|\mathcal{S}|^{2}+1$, which is independent of the number of nodes $n$ (i.e., $|\mathcal{S}|^{2}$ parameters correspond to the transition probability matrix $T_{\mathrm{m}}$ from Theorem 2 and one parameter corresponds to the credibility probability $q$ ).

The second approach is a direct (model-free) method that finds an $\varepsilon$-optimal solution of the Bellman equation 22 without learning the model. In this approach, any approximate dynamic programming method such as $\operatorname{TD}(\lambda)$ and Q-learning can be employed to find a sufficiently close approximation to the Bellman equation (22). To this end, it is important to note that the state space of Bellman equation 22 is finite and its states are observable by virtue of a model-independent function $\tilde{f}$ given by $(36)$. To illustrate the approach, we use the Qlearning algorithm in this paper as a model-free reinforcement learning method. We present Algorithm 1 which requires only $|\mathcal{M}(n)|\left|\mathbb{N}_{k}^{*}\right||\mathcal{A}|$ scalars for the Q-functions, where $\left|\mathbb{N}_{k}^{*}\right||\mathcal{A}|$ is, in fact, equal to $2(k+1)$.

Theorem 5. Let Assumption 11 hold, and suppose that every pair of state $(x, y) \in \mathcal{M}(n) \times \mathbb{N}_{k}^{*}$ and action $a \in \mathcal{A}$ is visited infinitely often in Algorithm 1 . Then, the following results hold:

(a) For any $(x, y, a) \in \mathcal{M}(n) \times \mathbb{N}_{k}^{*} \times \mathcal{A}$, the $Q$-function $Q(x, y, a)$ converges to $Q^{*}(x, y, a)$ with probability one.

(b) Let $g_{\varepsilon}^{*} \in \operatorname{argmin}_{a \in \mathcal{A}} Q^{*}(x, y, a)$ be a greedy strategy; then, $g_{\varepsilon}^{*}$ is an $\varepsilon$-optimal strategy for Problem 2 .

Proof. The proof is presented in Appendix E

\section{Networks With a Large Number of Nodes: ASYMPTOTIC ANALYSIS}

In this section, we show that estimating data tends to be more efficient than collecting it when the number of nodes is sufficiently large. To this end, the following assumption is made on the model.

Assumption 3. There exist positive scalars $K_{T}$ and $K_{c}$ such that for any $s^{\prime}, s \in \mathcal{S}, a \in \mathcal{A}$, and $m, m^{\prime} \in \mathcal{P}(\mathcal{S})$,

1. $\left|T\left(s^{\prime}, s, m\right)-T\left(s^{\prime}, s, m^{\prime}\right)\right| \leq K_{T}\left\|m-m^{\prime}\right\|$,

2. $c(m, \hat{m}, a) \leq K_{c}\|m-\hat{m}\|$.

It is to be noted that Assumption 3 is mild because any polynomial function in $m$ is Lipschitz with respect to $m$ due to the fact that $m$ is confined to a bounded interval. Let $\bar{T}$ : $\mathcal{P}(\mathcal{S}) \rightarrow \mathcal{P}(\mathcal{S})$ be defined as follows:

$$
\bar{T}(p):=\sum_{s \in \mathcal{S}} p(s) T(\cdot, s, p), \quad p \in \mathcal{P}(\mathcal{S}) .
$$

Let also the infinite-population estimator $h$ be defined as:

$$
\hat{m}_{t}=\underbrace{\bar{T} \circ \ldots \circ \bar{T}}_{t-1}\left(\hat{m}_{1}\right), \quad t \in \mathbb{N} \backslash\{1\},
$$

where $\circ$ is the composition operator.

Remark 4. When the dynamics are decoupled and $n=\infty$, the nonlinear dynamics (24) reduces to a linear equation, i.e., $\bar{T}(p)=T(\cdot, \cdot) p$.

Lemma 4. Let Assumption 3 hold. There exists a positive scalar $K_{p}$ such that

$$
\|\bar{T}(p)-\bar{T}(\hat{p})\| \leq K_{p}\|p-\hat{p}\|, \quad p, \hat{p} \in \mathcal{P}(\mathcal{S}) .
$$

Proof. The proof follows from equation 25, Assumption 3 , and the fact that the Lipschitz property is preserved under summation and multiplication.

Lemma 5. Let Assumption 2 hold. Given any $m_{t} \in \mathcal{M}(n)$ and $\hat{m}_{t} \in \mathcal{P}(\mathcal{S}), t \in \mathbb{N}$, the following inequality is satisfied:

$$
\mathbb{E}\left[\left\|m_{t+1}-\hat{m}_{t+1}\right\| \mid m_{t}, \hat{m}_{t}\right] \leq K_{p}\left\|m_{t}-\hat{m}_{t}\right\|+\mathcal{O}\left(\frac{1}{\sqrt{n}}\right) .
$$

Proof. The proof follows directly from the triangle inequality, Lemma 4 and [29, Lemma 2].

Assumption 4. Assume that $\gamma K_{p}<1$. Note that this inequality always holds when the dynamics of states in (2) are decoupled because in this case $K_{p}=1$ satisfies Lemma 4

Theorem 6. Let Assumptions 2, 3 and 4 hold. The total expected discounted cost associated with using the estimator 25. is bounded at all times and converges to zero at the rate $1 / \sqrt{n}$ as follows:

$$
\mathbb{E}\left[\sum_{t=1}^{\infty} \gamma^{t-1} c\left(m_{t}, \hat{m}_{t}, 0\right)\right] \leq \frac{\gamma K_{c}}{(1-\gamma)\left(1-\gamma K_{p}\right)} \mathcal{O}\left(\frac{1}{\sqrt{n}}\right),
$$

where $\mathcal{O}\left(\frac{1}{\sqrt{n}}\right)$ depends on the variance of local noises.

Proof. The proof is presented in Appendix F

Definition 1 (Certainty Threshold). The right-hand side of (26) is defined as the certainty threshold. This threshold 
depends on the number of nodes as well as the Lipschitz constants of the transition probability matrix and cost function introduced in Assumption 3

Corollary 3. Let Assumptions 2,4 hold. If the collection cost is greater than the certainty threshold, then the optimal solution to Problem 1 is to use estimator (25) at all times.

Proof. Consider two scenarios, where in the first one the strategy is to always use the estimator function (25), and in the second one the strategy is to collect data at least once. The costs of both scenarios until the first data collection are the same. The proof now follows directly from Theorem 6 .

Remark 5 (De Finetti's theorem). It is worth mentioning that when $n=\infty$, exchangeable random variables behave as i.i.d. variables, according to de Finetti's theorem [30].

\section{LINEAR DYNAMICS: A SPECIAL CASE}

\section{A. Optimal estimator}

The model presented in Subsection II-A has various applications, e.g., in remote-state estimation wherein an encoder sends a Markovian process to a decoder over an unreliable link under the Transmission Control Protocol (TCP), where an acknowledgement is sent to the encoder upon receiving the data at the decoder [31, 32]. The objective of the encoder and decoder is to collaborate in such a way that the cost function $[10]$ is minimized, where $g$ is the transmission strategy of the encoder and $h$ is the estimation strategy of the decoder. By using majorization theory and imposing some conditions such as symmetric and unimodal probability distribution of random variables, it is shown in [31, 32] that the optimal estimator is Kalman-like. In what follows, we extend the above findings by a simple proof technique using the proposed planning space, and subsequently establish a separation theorem that holds for the general case of multi-dimensional dynamic systems with an arbitrary probability distribution, without resorting to majorization theory or any other conditions on the random variables. To this end, define $x_{t} \in \mathbb{R}^{d_{s}}$ as the last (credible) observed data and $y_{t} \in \mathbb{N}^{*}$ as the elapsed time since then.

Theorem 7 (Separation principle). The problem of finding the optimal estimator in (10) is separated from that of the optimal scheduling strategy. In particular, the optimal estimator has a structure similar to the minimum mean-square estimator with the following Kamlan-like update rule:

$$
\hat{m}_{t+1}=A \hat{m}_{t}+L\left(y_{t+1}\right)\left(x_{t+1}-A \hat{m}_{t}\right),
$$

where $L\left(y_{t+1}\right)=1$ if $y_{t+1}=0$ and $L\left(y_{t+1}\right)=0$ if $y_{t+1} \neq 0$. This result holds regardless of the sampling strategy, the order of dynamics, the probability distribution of the initial states, credibility of data and additive noises.

Proof. The proof is presented in Appendix G

Remark 6. The result of Theorem 7 also holds for the timevarying finite-horizon case since the proof technique presented above does not depend on the time-homogeneity of the model.

\section{B. Sampling (scheduling) strategy}

Consider a centralized networked control system wherein the joint state is perfectly observed and the optimal joint action is a state-feedback strategy. In such a case, every node must broadcast its state at each time instant so that all nodes can observe the joint state in order to compute their control actions accordingly. In practice, however, sharing information is costly, the quality of data transmission is sometimes compromised, and data packets may be lost in the communication channels (e.g., in erasure channels). Thus, it is important to be able to implement the centralized solution in a distributed manner. To this end, each node can solve a (local) scheduling problem in order to decide when to broadcast its state [33]. Once the information is broadcast, other nodes can update their estimates of the state of the node, to be used in their strategies. For the case where every node uses a minimum mean-square estimator and attention is restricted to a threshold-type scheduling, a dynamic-program-based solution can be developed whose information state is the estimation error (that is a continuous variable in $\mathbb{R}^{d_{s}}$ with a modeldependent dynamics) [33]. It is shown in [31, 32] that such threshold-type policies are optimal under certain conditions.

In this work, we use a dynamic program with an information state different from [31-33], which is a discrete variable between 0 and $k$, independent of the state space dimension $d_{s}$, with a model-free dynamics. Therefore, it is computationally easy to find a near-optimal strategy by solving our proposed dynamic program.

Assumption 5. Suppose that the per-step cost (10) satisfies the inequality $c_{t}\left(m_{t}-\hat{m}_{t}, a_{t}\right) \leq c_{\max }$, where $c_{\max }$ is a known positive constant and the estimator is the minimum meansquare estimator, i.e., $\hat{m}_{t}=\mathbb{E}\left[m_{t} \mid o_{1: t}, a_{1: t-1}\right], t \in \mathbb{N}$.

From Assumption 5 and the dynamics of the mean-square estimator given by $(27)$, it follows that for any $t \in \mathbb{N}$ :

$$
m_{t}-\hat{m}_{t}=\mathbb{1}\left(y_{t}>0\right) \sum_{\tau=1}^{y_{t}} A^{\tau-1} \bar{w}_{t-\tau} .
$$

Since $w_{1: \infty}$ is an i.i.d. random process, one has:

$$
c_{t}\left(y_{t}, a_{t}\right)=\mathbb{E}\left[c_{t}\left(m_{t}-\hat{m}_{t}, a_{t}\right) \mid x_{1: t}, y_{1: t}, a_{1: t}\right],
$$

where the above per-step cost does not depend on $x_{t}$; hence, it can be denoted by $c\left(y_{t}, a_{t}\right), t \in \mathbb{N}$. It is also possible to consider a special case of Assumption 5, described below, that provides an explicit expression for (28).

Assumption 6. For any $t \in \mathbb{N}, a_{t} \in \mathcal{A}$ and $m_{t} \in \mathcal{M}(n)$, let functions $z\left(a_{t}\right)$ and $\ell\left(m_{t}, a_{t}\right)$ in 10 be equal to 1 and $\ell a_{t}$, respectively, where $\ell \in \mathbb{R}_{\geq 0}$. In addition, matrix $A$ is symmetric and all of its eigenvalues are within the unit circle. ${ }^{4}$

\footnotetext{
${ }^{4}$ For finite-horizon analysis, $A$ can be any arbitrary matrix.
} 
Under Assumption 6 and Theorem 7, (28) can be computed as follows:

$$
\begin{aligned}
c\left(y_{t}, a_{t}\right) & =\mathbb{E}\left[\left(m_{t}-\hat{m}_{t}\right)^{\top}\left(m_{t}-\hat{m}_{t}\right)+\ell a_{t} \mid x_{1: t}, y_{1: t}, a_{1: t}\right] \\
= & \mathbb{1}\left(y_{t}>0\right) \operatorname{Tr}\left(\sum_{\tau=1}^{y_{t}}\left(A^{\top} A\right)^{\tau-1} \bar{\Sigma}^{w}\right)+\ell a_{t}, \\
= & \operatorname{Tr}\left(\left(\mathbf{I}-A^{\top} A\right)^{-1}\left(\mathbf{I}-\left(A^{\top} A\right)^{y_{t}}\right) \bar{\Sigma}^{w}\right)+\ell a_{t} .
\end{aligned}
$$

where $\mathbf{I}$ is the identity matrix and $\bar{\Sigma}^{w}$ is the covariance matrix of $\bar{w}_{t}, t \in \mathbb{N}$. The following theorem is a consequence of Theorems 4 and 5.

Theorem 8 (Sampling strategy). Let either Assumption 5 or Assumption 6 hold. There is no loss of optimality in restricting attention to the space of elapsed times after the last credible data (i.e., there is no need to know the data). More precisely, select a sufficiently large $k \in \mathbb{N}$ such that inequality (23) is satisfied, and simplify the dynamic program (22) as follows:

$$
\tilde{V}_{k}(y)=\min \left(\tilde{V}_{k}^{0}(y), \tilde{V}_{k}^{1}(y)\right), \quad y \in \mathbb{N}_{k}^{*},
$$

where $\tilde{V}_{k}^{0}(y):=c(y, 0)+\gamma\left(\mathbb{1}(y<k) \tilde{V}_{k}(y+1)+\mathbb{1}(y=\right.$ $\left.k) \tilde{V}_{k}(0)\right)$ and $\tilde{V}_{k}^{1}(y):=c(y, 1)+(1-q) \gamma\left(\mathbb{1}(y<k) \tilde{V}_{k}(y+1)\right.$ $\left.+\mathbb{1}(y=k) \tilde{V}_{k}(0)\right)+q \gamma \tilde{V}_{k}(0)$. Then, a near-optimal sampling strategy is to collect data when $\tilde{V}_{k}^{0}\left(y_{t}\right)<\tilde{V}_{k}^{1}\left(y_{t}\right)$. A similar relationship holds for the case that model structure is not known completely, where Q-learning algorithm proposed in Algorithm 1 converges to a near-optimal solution.

Proof. The proof follows form the fact that the per-step cost in (28) and (29) does not depend on $x_{t}$; hence, $x_{t}$ is irrelevant information.

Corollary 4. Let Assumptions 2,3 and 6 hold. The certainty threshold in the case of linear dynamics with quadratic cost function converges to zero at the rate $1 / n$, which is faster than the generic rate of $1 / \sqrt{n}$. In this case, $\bar{\Sigma}^{w}=$ $\frac{1}{n} \operatorname{diag}\left(\Sigma^{w}, \ldots, \Sigma^{w}\right)$, where $\Sigma^{w}$ is the covariance matrix of an individual local noise.

Remark 7 (Time delay). All the results presented in this paper, including Theorems 7 and 8 , extend naturally to the case where observations are received with a fixed time delay $\tau \in \mathbb{N}^{*}$, by simply replacing $y_{t}$ with $y_{t}+\tau$.

Remark 8 (Mean-field approximation). When $n=\infty$ and the dynamics are decoupled, the infinite-population (linear) model presented in Remark 4 may be used in Theorem 8 to provide a scale-free approximation.

Remark 9 (Age of information). Note that the cost function (29) is exponential with respect to $y_{t}$, reflecting the fact that the quality of the minimum mean-square estimator deteriorates exponentially in the absence of credible data. Nonetheless, it is possible to consider a simpler cost function, e.g., an affine or quadratic cost function in $y_{t}$, for which the minimum of the right-hand side of the dynamic program 30 can be obtained more efficiently. This case is then related to real-time status updating, where $y_{t}$ is the age of information, representing the freshness of data, and the objective is to monitor a phenomenon of interest in a timely manner [34-
36]. Hence, the dynamic program (30) and its reinforcement learning version can be used to determine a low-complexity near-optimal solution for minimizing the age of information.

\section{Noisy observations with Gaussian random variables}

In this subsection, we show that the presence of measurement noise adversely impacts the tractability gained by the proposed planning space, and consequently finding an $\varepsilon$ optimal solution becomes NP-hard. However, we demonstrate that the resultant optimization problem is a deterministic nonlinear dynamic optimization problem that may be solved numerically by various computational tools. Suppose that Assumption 6 holds, and that local noises are Gaussian. Let $o_{t}^{i} \in \mathbb{R}^{d_{o}}, d_{o} \in \mathbb{N}$, be the noisy observation of node $i$ at time $t$, i.e., $o_{t}^{i}=C s_{t}^{i}+\xi_{t}^{i}$, where $\xi_{1: \infty}^{i}$ is an i.i.d. Gaussian random process with zero mean and finite covariance matrix $\Sigma^{i, \xi} \in \mathbb{R}^{d_{o} \times d_{o}}$. In addition, it is assumed that the measurement noises $\left\{\xi_{1: \infty}^{i}\right\}_{i \in \mathbb{N}_{n}}$ are mutually independent across nodes, and are also independent from the previously defined primitive random variables. Then,

$$
\bar{o}_{t}^{d}:=\frac{1}{n} \sum_{i=1}^{n} v^{i, d} o_{t}^{i}=C_{d} m_{t}^{d}+\bar{\xi}_{t}^{d},
$$

where $C_{d}:=\frac{1}{n} \sum_{i=1}^{n} v^{i, d} C$ and $\bar{\xi}_{t}^{d}:=\frac{1}{n} \sum_{i=1}^{n} v^{i, d} \xi_{t}^{i}$ with the covariance matrix $\sum^{\xi, d}:=\frac{1}{n^{2}} \sum_{i=1}^{n}\left(v^{i, d}\right)^{2} \sum^{i, \xi}$. For simplicity of presentation, assume that $q=1$, and that the horizon is finite. Therefore,

$$
o_{t+1}:=C\left(a_{t}\right) m_{t+1}+E\left(a_{t}\right) \bar{\xi}_{t+1},
$$

where $o_{t}=\operatorname{vec}\left(o_{t}^{1}, \ldots, o_{t}^{D}\right), C\left(a_{t}\right):=a_{t} \operatorname{diag}\left(C_{1}, \ldots, C_{D}\right)$, $\bar{\xi}_{t}=\operatorname{vec}\left(\bar{\xi}_{t}^{1}, \ldots, \bar{\xi}_{t}^{D}\right), \bar{\Sigma}^{\xi}=\operatorname{diag}\left(\bar{\Sigma}^{\xi, 1}, \ldots, \bar{\Sigma}^{\xi, D}\right)$ and $E\left(a_{t}\right)=a_{t}$. In general, blank observation does not carry the same information that zero observation does. However, when attention is restricted to Gaussian random variables, the conditional expectation of the state, given zero observation, is equal to that given the blank observation because the innovation processes associated with both cases are zero. Subsequently, from [37], one can use the celebrated Kalman filter to compute the optimal state estimate from noisy observations. In particular, given any realization $a_{1: t}$, the minimization in (10) reduces to a mean-square optimization problem, where the best nonlinear estimator is known to be $\hat{m}_{t}=\mathbb{E}\left[m_{t} \mid o_{1: t}, a_{1: t-1}\right]$. In a way similar to [37], define the following covariance matrix:

$$
\begin{aligned}
P_{t+1}= & A P_{t} A^{\boldsymbol{\top}}+\bar{\Sigma}^{w}-A P_{t} C^{\boldsymbol{\top}}\left(a_{t}\right)\left(C\left(a_{t}\right) P_{t} C^{\boldsymbol{\top}}\left(a_{t}\right)\right. \\
& \left.+E\left(a_{t}\right) \bar{\Sigma}^{\xi} E^{\boldsymbol{\top}}\left(a_{t}\right)\right)^{-1} C\left(a_{t}\right) P_{t} A^{\boldsymbol{\top}}, \quad t \in \mathbb{N},
\end{aligned}
$$

where $P_{1}=\mathbf{0}_{D d_{s} \times D d_{s}}$. Then, the optimal estimator is given by the following Kalman filter:

$$
\hat{m}_{t+1}=A \hat{m}_{t}+L\left(a_{t}\right)\left(o_{t+1}-C\left(a_{t}\right) A \hat{m}_{t}\right),
$$

where $\hat{m}_{1}=\mathbf{0}_{D d_{s} \times 1}$ and the observer gain is described by:

$L\left(a_{t}\right)=\left(A P_{t} C^{\boldsymbol{\top}}\left(a_{t}\right)\right)\left(C\left(a_{t}\right) P_{t} C^{\boldsymbol{\top}}\left(a_{t}\right)+E\left(a_{t}\right) \bar{\Sigma}^{\xi} E^{\boldsymbol{\top}}\left(a_{t}\right)\right)^{-1}$.

Consequently, the optimization problem associated with the optimal scheduling strategy for any finite horizon $H$ reduces 
to a deterministic non-convex nonlinear optimization problem as follows:

$$
\min _{a_{1: H}} \sum_{t=1}^{H} \gamma^{t-1}\left(\operatorname{Tr}\left(P_{t+1}\right)+\ell a_{t}\right)
$$

To find a solution to the above optimization problem, one can construct a dynamic program based on the history space $\left\{a_{1: H}\right\}$, whose cardinality grows exponentially with the horizon (i.e. $2^{H}$ ). Alternatively, one can write a dynamic program based on the information state $P_{t}$ (which is a continuous variable in $\mathbb{R}^{D d_{s}} \times \mathbb{R}^{D d_{s}}$ with nonlinear model-dependent dynamics (31)). For a reasonably large horizon $H$, both dynamic programs can be very difficult to solve analytically.

\section{Generalization to COMPlex Networks}

The main focus of the previous sections was to study the trade-off between data collection and data estimation, and for this reason, the simplest model structure was considered in order for the excessive number of parameters not to obscure the main results. In this section, we show how our results can naturally be extended to more complex applications.

\section{A. Multiple decision makers and estimators}

Consider a network with $\tilde{n} \in \mathbb{N}$ decision makers, and let $\hat{n}(k) \in \mathbb{N}$ denote the number of estimators whose access to data is decided by decision maker $k \in \mathbb{N}_{\tilde{n}}$. In such a setup, for any $j \in \mathbb{N}_{\hat{n}(k)}$, estimator $j$ provides a different estimate $\hat{m}^{k, j}$ of the states of all nodes. Thus, the objective is to minimize the following social cost function:

$$
\mathbb{E}^{g}\left[\sum_{t=1}^{\infty} \gamma^{t-1} \sum_{k=1}^{\tilde{n}} c^{k}\left(m_{t}, \hat{m}_{t}^{k, 1}, \ldots, \hat{m}^{k, \hat{n}(k)}, a_{t}^{k}\right)\right] .
$$

Since the state dynamics is not influenced by the actions of decision makers, and on the other hand, the above cost function is additive, the optimization problem of each decision maker (i.e., Problems 1 and 2) can be solved separately, with possibly different parameters. Therefore, the proposed concept of planning space is applicable here.

\section{B. Multiple reset actions}

So far, the trade-off between data collection and data estimation has been formulated as a binary decision, where $a_{t}=0$ means that the data are not collected and $a_{t}=1$ means that the data are collected (note that the collected data are not necessarily credible). It is possible to generalize the above decision to multiple decision options, which correspond to, for example, using different routes, channels, sensors and receivers. To this end, it is required to define the last credible data $x_{t}^{i}$ and elapsed time $y_{t}^{i}$ for each option $i \in \mathbb{N}$ so that when the credible data $x_{t}^{i}$ are received at a particular time $t$ via the $i$-th option, its elapsed time resets to zero (i.e., $y_{t}^{i}=0$ ) [21, Remark 2]. This extension is similar to a bandit problem, where each option represents a bandit. Consequently, an immediate application of the data collection/estimation analysis is to address the tradeoff between exploration and exploitation that arises in various learning tasks, where a decision maker wishes to sequentially choose when to explore (collect the data of interest) and when to exploit the learned model (which is data estimation based on the previously collected data). For multiple reset actions, see an example of machine maintenance problem with three actions in [38].

\section{Partially exchangeable and partially equivariant networks}

Consider partially exchangeable networks for the Markovchain model and partially equivariant networks for the linear model [3, 4]. The population of nodes is partitioned into a few sub-populations, some with Markov-chain model and some with linear model, as described above, wherein the nodes in the former sub-population are exchangeable and the ones in the latter one are equivariant. In this case, the data dynamics becomes more complex but the proposed approach still works because the dynamics does not depend on the action of the decision maker. For an example of partially exchangeable network, see a leader-follower network in [39] with $n$ exchangeable followers and one non-exchangeable leader.

\section{Markovian noise and credibility processes}

Depending on the data (state of the system), Assumptions 1 and 2 can be generalized to the case in which local noises have their own Markov-chain dynamics. In such a case, dynamic programming decomposition is still valid, with the only difference that the state of the Markov chain is added to the system state. Note that Assumptions 1 and 2 are not required for the linear case (i.e., Theorems 7 and 8). Similarly, credibility can be a Markovian process, e.g., see [40] for the spread of fake news in a homogeneous network.

\section{APPLICATIONS}

\section{A. A sensor network with prioritized data}

We consider a sensor (decision maker) that measures a Markovian source $s_{t}$ at time $t \in \mathbb{N}$, such as the temperature of a room or the battery charge state of a smart house, and wishes to report it to a data center. Let $\mathcal{S}:=\mathbb{Z}_{d_{s}+d_{w}}, d_{s}, d_{w} \in \mathbb{N}$, be the state space, and $s_{t}$ evolve in time as follows:

$$
s_{t+1}= \begin{cases}s_{\max }, & s_{t}>d_{s}, \\ s_{t}+w_{t}, & \left|s_{t}\right| \leq d_{s}, \\ s_{\min }, & s_{t}<-d_{s},\end{cases}
$$

where $s_{\max }, s_{\min } \in \mathcal{S}$ are the saturation levels, and for any $t \in \mathbb{N}, w_{t} \in \mathcal{W}:=\mathbb{Z}_{d_{w}}$ is an i.i.d. process with probability distribution function $P_{W}$. The state $s_{t+1}$ is successfully received at the data center upon transmission (i.e., $a_{t}=1$ ) with probability $q \in[0,1]$ at time $t \in \mathbb{N}$, i.e., for any $s \in \mathcal{S}$, $\mathbb{P}\left(o_{t+1}=s \mid s_{t+1}=s, a_{t}=1\right)=q$. In practice, it is not efficient for the sensor to measure $s_{t}$ and transmit it to the data center at each time instant $t \in \mathbb{N}$ because there is often a cost associated with sensing and transmitting. Let $\hat{s}_{t} \in \mathcal{S}$ be the last state received by time $t \in \mathbb{N}$ at the data center. The objective of the sensor is to find an efficient transmission law that not only keeps the estimation error small at the data 
center, but also incurs minimal measurement and transmission cost at the sensor. The following performance index is defined:

$$
J=\mathbb{E}\left[\sum_{t=1}^{\infty} \gamma^{t-1}\left(\left|s_{t}\right|\left|s_{t}-\hat{s}_{t}\right|+\ell a_{t}\right)\right],
$$

where $\gamma \in(0,1)$ is the discount factor and $\ell \in \mathbb{R}_{>0}$ is the transmission cost. Note that the estimation error $\left|s_{t}-\hat{s}_{t}\right|$ in the above cost function is weighted by $\left|s_{t}\right|$; the rationale for using such a penalty term lies in the fact that in some applications the saturation levels represent warning zones, in the sense that the estimation error around such zones carries more weight than that around normal operating zones: hence, classical thresholdbased strategies that treat all states equally are not practical.

Example 1. Suppose that $s_{t}$ is the energy level of a battery. The battery is charged by some renewable generation sources and discharged as serving demands. Initially, the nominal value of the battery is $s_{1}=0$. At each time $t \in \mathbb{N}$, one unit energy is added to $s_{t}$ with probability $p_{g}$ and one unit energy is depleted from $s_{t}$ with probability $p_{d}$, where the probability of the generation $p_{g}$ is independent of that of the consumption $p_{d}$. Let $w_{t} \in\{-1,0,1\}$ denote the change in the energy level of the battery at time $t$, i.e., $P\left(w_{t}=1\right)=p_{g}\left(1-p_{d}\right), P\left(w_{t}=-1\right)=p_{d}\left(1-p_{g}\right)$, and $P\left(w_{t}=0\right)=p_{g} \times p_{d}+\left(1-p_{g}\right) \times\left(1-p_{d}\right)$. The objective is to find the optimal frequency for transmitting the state of the battery under the transmission cost $\ell$. Let $p_{g}=p_{d}=0.8$, $d_{w}=1, d_{s}=s_{\max }=-s_{\min }=99, q=0.95, \gamma=0.9$, and $\ell=100$. Due to the incompleteness of the information structure at the decision making level, the conventional belief space is large: more precisely, $\mathbb{P}\left(s_{t} \mid o_{1: t}\right) \in \mathbb{R}^{200}$. In addition, reinforcement learning in belief space is conceptually difficult because the dynamics of the belief state depends on the model (i.e., it is a model-dependent planning space). Thus, we use a new information state based on which the proposed strategies in both planning and reinforcement learning cases are tractable, and their performances are sufficiently close to the optimal performance. The number of states in the new planning space is $10^{3}=200 \times 50$.

Let $x_{t} \in \mathcal{S}$ denote the last credible observation of the data center by time $t \in \mathbb{N}$ (i.e., $x_{t}=\hat{s}_{t}$ ) and $y_{t} \in \mathbb{N}^{*}$ denote the elapsed time after receiving $x_{t}$. A near-optimal transmission law can be obtained by solving the Bellman equation 22. in Theorem 4 for a sufficiently large approximation index $k \in \mathbb{N}$, where the space $\mathcal{M}(n)$ and transition probability matrix $T_{\mathrm{m}}$ are replaced by $\mathcal{S}$ and $T$, respectively, according to Corollary 2 After exhaustive simulations, it is observed that the optimal strategy is obtained for any approximation index $k \geq 705$ According to Figure 1, the frequency of transmitting the energy level of the battery to the data center increases as the energy level approaches the warning thresholds. When the generation probability $p_{g}$, consumption probability $p_{d}$, and successful delivery probability $q$ are all unknown, one can use Q-learning algorithm (Algorithm 1) to obtain the optimal solution. For the purpose of display, the convergence of the

${ }^{5}$ For $\epsilon=10^{-3}$, inequality 23 holds for any $k \geq 189$. On the other hand, it is observed in simulations that the optimal strategy is obtained for any $k \geq 70$.

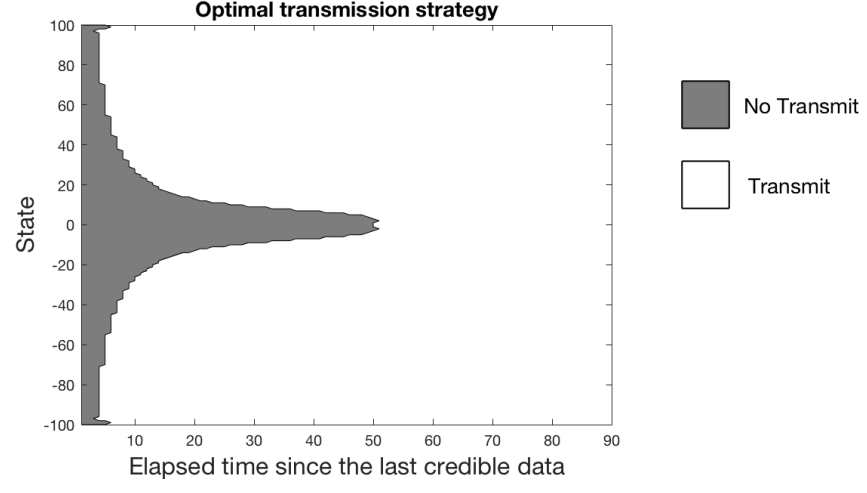

Fig. 1. Optimal transmission strategy in Example 1.

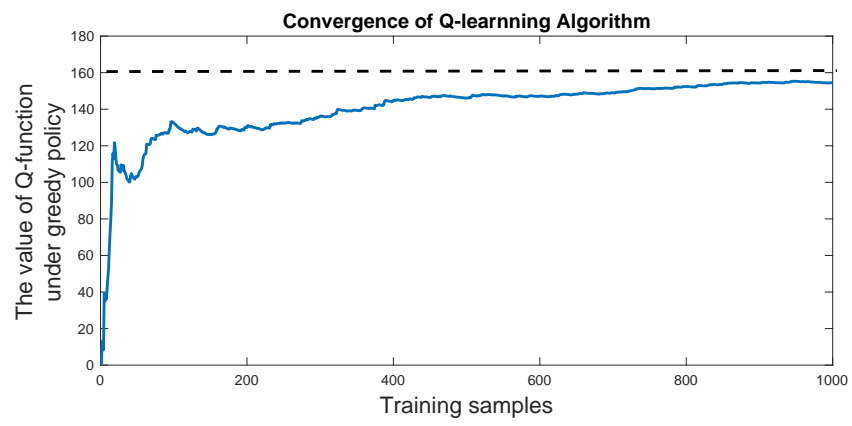

Fig. 2. The convergence of the Q-learning algorithm (Algorithm 1 at state $(x, y)=(0,50)$ in Example 1 .

algorithm is depicted at state $(x, y)=(0,50)$ in Figure 2. It is shown that $\min _{a \in \mathcal{A}} Q(0,50, a)$ converges to the optimal value function $V(0,50)=160.83$. In this example, the Q-learning algorithm (Algorithm 1) is trained offline, where at each training sample a batch update is performed over the entire state-action pairs (Q-functions), also known as synchronized parallel Q-learning, with step sizes inversely proportional to the number of visits (updates) to each pair of state and action. On a Mac Pro laptop with $2.7 \mathrm{GHz}$ Intel Core i5, the algorithm with a known-model runs in 250 seconds, and with an unknown-model in 6 hours.

\section{B. A communication network with packet drop}

Inspired by recent developments in networked control systems [31, 33] and deep teams [3, 4, 39], we consider $n$ networked controllers that use a deep structured optimal statefeedback strategy. In this case, the dynamics of the deep state (weighted average of the states of the controllers) is in the form of (9). Now, consider an authority (e.g., an independent service operator in a smart grid) wishing to collect the deep state, transmit it over an unreliable channel, and eventually receive it at a decoder, located far away from the control site. The objective is to find an efficient way to construct a reliable estimate at the decoder with minimum possible collection cost, while taking into account the topology of the network and unreliability of the transmission (formulated as packet drop). A block diagram of the above system is displayed in Figure 3 
Example 2. Consider two topologies, a complete graph and a star graph, with the dominant eigenvalues $(n-1)$ and $\pm \sqrt{n-1}$, respectively. Suppose $L=1, \alpha(0)=0$, and $\alpha(1)=$ $\frac{A}{n-1}, A \in \mathbb{R}$, in the vectorized dynamics $[8]$, where $v^{C}=$ $\operatorname{vec}(1, \ldots, 1)$ and $v^{S}=\frac{\sqrt{n}}{\sqrt{2(n-1)}} \operatorname{vec}( \pm \sqrt{n-1}, 1, \ldots, 1)$ are the eigenvectors of the dominant eigenvalues of the complete and star graphs, respectively. Hence, the dynamics of the weighted average of the dominant mode of the complete graph is given by:

$$
m_{t+1}^{C}=A m_{t}^{C}+\bar{w}_{t}^{C},
$$

where $m_{t}^{C}=\frac{1}{n} \sum_{i=1}^{n} v^{c}(i) s_{t}^{i}=\frac{1}{n} \sum_{i=1}^{n} s_{t}^{i}$ and $\bar{w}_{t}^{C}=$ $\frac{1}{n} \sum_{i=1}^{n} w_{t}^{i}$. Similarly, the dynamics of the weighted average of the dominant mode of the star graph is described by:

$$
m_{t+1}^{S}=\frac{ \pm A}{\sqrt{n-1}} m_{t}^{S}+\bar{w}_{t}^{S},
$$

where $m_{t}^{S}=\frac{1}{n} \sum_{i=1}^{n} v^{S}(i) s_{t}^{i}=\frac{ \pm 1}{\sqrt{2 n}} s_{t}^{1}+\frac{1}{\sqrt{2 n(n-1)}} \sum_{i=2}^{n} s_{t}^{i}$ and $\bar{w}_{t}^{S}=\frac{ \pm 1}{\sqrt{2 n}} w_{t}^{1}+\frac{1}{\sqrt{2 n(n-1)}} \sum_{i=2}^{n} w_{t}^{i}$, with $s_{t}^{1}$ and $w_{t}^{1}$ denoting the state and local noise of the central node. The per-step cost function is given by (10) under Assumption 6 In addition, suppose that the probability of data being dropped is $1-q=0.1$ and the discount factor is $\gamma=0.85$. Local noises are i.i.d. random variables with zero mean and finite variance $\Sigma^{w}$ (that are not necessarily Gaussian or symmetric with a unimodal distribution). From Theorem 8 , one can find an optimal strategy for a sufficiently large approximation index $k \in \mathbb{N}$. The optimal sampling strategy is shown in Figure 4 with respect to the number of nodes $n$ and the variance of local noises $\Sigma^{w}$. In addition, the optimal estimate is constructed at the decoder based on the result of Theorem 7 It is observed from Figure 4 that the data must be sampled more frequently in the complete graph, as the variance of noise increases. This is not surprising because information flows faster in a complete graph. Furthermore, according to this figure, the certainty threshold of the complete graph is larger than that of the star graph, with respect to the number of nodes. In these simulations, the approximation index $k$ is set to 200, which guarantees $\epsilon$-optimality of the proposed strategies for any $\epsilon \geq 10^{-5}$.

In the case when collection cost $\ell$, packet-drop probability $1-q$, number of nodes $n$, and system matrices $A$ and $\Sigma^{w}$ are all unknown, one can use the RL algorithm proposed in Theorem 5 to obtain a near-optimal scheduling strategy. It is to be noted that the proposed RL is not applicable to the case where the topology is unknown because we still need to know the eigenvectors of the graph Laplacian for computing the data of interest.

\section{A social network with binary states}

Consider a social network consisting of $n$ users. Denote by $s_{t}^{i} \in \mathcal{S}$ the opinion of user $i \in \mathbb{N}_{n}$ at time $t \in \mathbb{N}$. Every user $i \in \mathbb{N}_{n}$ independently changes its opinion from $s_{t}^{i}$ to $s_{t+1}^{i}$ with probability $\mathbb{P}\left(s_{t+1}^{i} \mid s_{t}^{i}, m_{t}\right)$ at time $t$. An agency wants to conduct a survey from users to collect the percentage of their opinions and update it frequently; however, it is not practical (and is expensive) to conduct a survey at every time

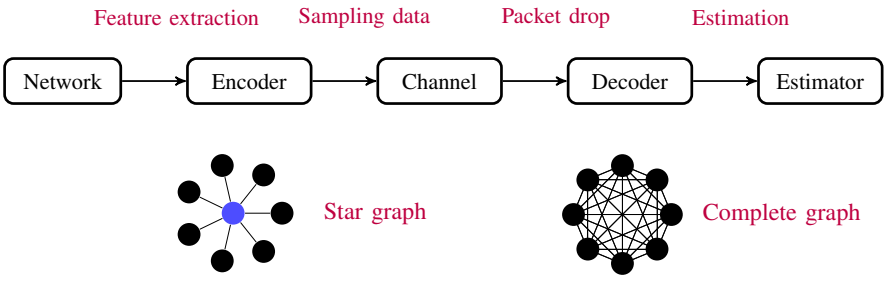

Fig. 3. The block diagram of the system in Example 2.

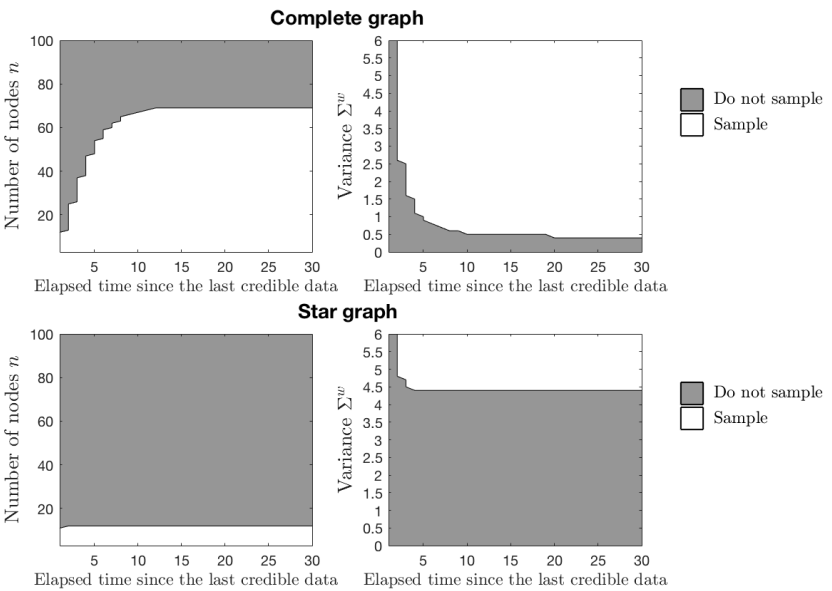

Fig. 4. Near-optimal strategies in Example 2. In the left-hand side plots the dependency of the strategies to the number of nodes and elapsed time is demonstrated, using the parameters: $A=0.8, \ell=0.4$ and $\Sigma^{w}=6$. In the right-hand side plots the dependency of the strategies to the variance of local noises and elapsed time is displayed, using parameters: $A=0.9, \ell=1$ and $n=5$.

step. Consequently, it is desirable to design a cost-effective yet informative strategy for conducting surveys.

Let $\ell \in \mathbb{R}_{>0}$ be the price of conducting a survey (e.g., operators' costs and also monetary rewards to incentivize the users to participate) and $q$ be the probability that the result of a survey is credible. If a survey result is not credible, it is thrown away. When there is no survey, the agency uses maximum a posteriori probability (MAP) estimator to estimate the percentage of opinions from its previously conducted surveys, i.e.,

$$
\hat{m}_{t}=\underset{m \in \mathcal{M}(n)}{\operatorname{argmax}}\left(\mathbb{P}\left(m_{t}=m \mid o_{1: t}, a_{1: t}\right)\right) .
$$

Given a discount factor $\gamma \in(0,1)$, the objective is to minimize the following expected total discounted cost:

$$
J=\mathbb{E}\left[\sum_{t=1}^{\infty} \gamma^{t-1}\left(D_{\mathrm{KL}}\left(m_{t} \| \hat{m}_{t}\right)+\ell a_{t}\right)\right],
$$

where $D_{\mathrm{KL}}\left(m_{t} \| \hat{m}_{t}\right)=\sum_{s \in \mathcal{S}} m_{t}(s) \log \left(\frac{m_{t}(s)}{\hat{m}_{t}(s)}\right)$ denotes the Kullback-Leibler divergence. At any time $t \in \mathbb{N}$, let $x_{t} \in$ $\mathcal{M}(n)$ be the empirical distribution of the opinions collected at the last survey and $y_{t} \in \mathbb{N}^{*}$ be the elapsed time after the last survey. From (18) and (32, $\hat{m}_{t}=$ $\operatorname{argmax}_{m \in \mathcal{M}(n)} T_{\mathrm{m}}^{y_{t}}\left(m, x_{t}\right)$, where the transition probability matrix $T_{\mathrm{m}}$ is given by Theorem 11. A near-optimal strategy can be obtained by Theorem 4 for a sufficiently large $k$. 


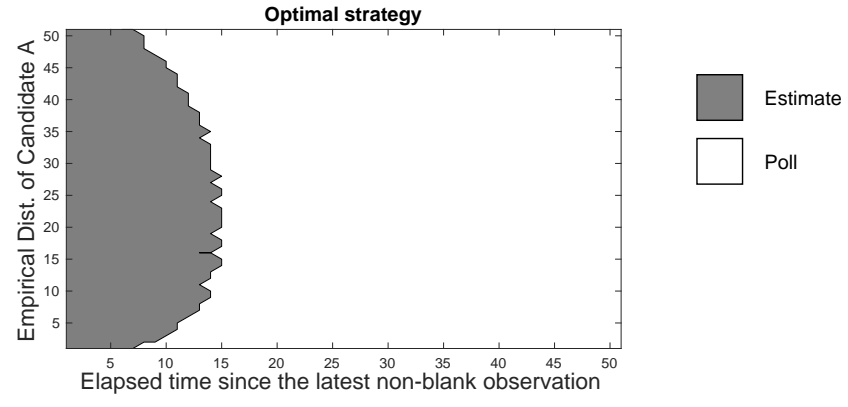

Fig. 5. Optimal strategy for conducting polls in Example 3. The unsmooth surface of the solution is due to the fact that the MAP estimator is not smooth.

Example 3. Consider an election between two candidates $A$ and $B$, i.e., $\mathcal{S}=\{A, B\}$. An agency is interested to conduct polls among $n \in \mathbb{N}$ voters. With a slight abuse of notation, let $m_{t}:=m_{t}(A) \in\left\{0, \frac{1}{n}, \frac{2}{n}, \ldots, 1\right\}$ represent the empirical distribution of the voters who prefer candidate $A$ at time $t \in \mathbb{N}$. Since the state space $\mathcal{S}$ is binary, $m_{t}$ is sufficient for determining the empirical distribution of the voters favoring candidate $B$ which is $1-m_{t}$. Note that the empirical distribution $m_{t}$ takes $n+1$ different values, and its belief state is $\mathbb{P}\left(m_{t} \mid o_{1: t}\right) \in \mathbb{R}^{n+1}$. In contrast, our proposed planning space is a discrete space with $(n+1) \times k$ values, which is a considerable reduction with respect to $n$. Let the number of voters be $n=50$. In addition, let $\mathbb{P}\left(s_{t+1}^{i}=A \mid s_{t}^{i}=A\right)=0.95$, i.e., the probability that voter $i \in \mathbb{N}_{n}$ chooses candidate $A$ at the next time instant if this is currently the voter's favorite candidate. Similarly, $\mathbb{P}\left(s_{t+1}^{i}=B \mid s_{t}^{i}=B\right)=0.98$, i.e., the probability that voter $i$ chooses candidate $B$ at the next time instant if $B$ is the voter's current choice. Suppose that the cost of running a poll is $\ell=0.02$, the discount factor is $\gamma=0.8$, and the probability of the credibility of a poll is $q=0.95$. Exhaustive simulations demonstrate that the optimal solution is attained for any approximation index $k \geq 50^{6}$ The optimal strategy is displayed in Figure 5, which demonstrates that when the number of voters in favor of candidate $A$ is 45 , the agency should run a poll after the elapsed time from the latest credible observation exceeds 10 . When, on the other hand, the number of voters in favor of candidate $B$ is 45 , the agency should wait slightly longer (11 time instants) before conducting a new poll. This difference is due to the fact that voters are more likely to change their opinion if candidate $A$ is their current choice. The simulation time is 116 seconds on a computer with the specification described in Example 1.

\section{CONCLUSiOnS}

In this paper, the trade-off between data collection and estimation in networks with both known and unknown models was investigated. Some important properties of the dynamics of data were studied first, and an $\varepsilon$-optimal solution was subsequently provided using the Bellman equation. The proposed solution was then extended to the case where the model is not completely known, using two different learning-based

\footnotetext{
${ }^{6}$ For $\epsilon=10^{-5}$, inequality 23 holds for any $k \geq 50$.
}

approaches. It was also shown at what point estimating data tends to be more desirable than collecting data as the number of nodes increases. The special case of linear dynamics was studied in more detail, and a separation principle was presented accordingly. Three numerical examples were provided to demonstrate the effectiveness of the proposed strategies.

For future research directions, it would be interesting to study the computational complexity of the proposed approach under various approximation methods such as stochastic approximation, linearization, particle filtering, quantization, randomization, and Monte-Carlo simulation as well as different simplifying, yet realistic, assumptions such as ergodicity and the myopicity of the decision process. In particular, the reader is referred to [26] and references therein for various stochastic numerical methods that can be used for more efficient computation, albeit at the cost of losing the performance guarantee.

\section{REFERENCES}

[1] Y. LeCun, Y. Bengio, and G. Hinton, "Deep learning," nature, vol. 521, no. 7553, pp. 436-444, 2015.

[2] J. Schmidhuber, "Deep learning in neural networks: An overview," Neural networks, vol. 61, pp. 85-117, 2015.

[3] J. Arabneydi and A. G. Aghdam, "Deep teams: Decentralized decision making with finite and infinite number of agents," IEEE Transactions on Automatic Control, vol. 65, no. 10, pp. 4230-4245, 2020.

[4] J. Arabneydi and A. G. Aghdam, "Deep structured teams with linear quadratic model: Partial equivariance and gauge transformation," [Online]. Available at https://arxiv.org/abs/1912.03951, 2019.

[5] A. Jadbabaie, P. Molavi, A. Sandroni, and A. TahbazSalehi, "Non-bayesian social learning," Games and Economic Behavior, vol. 76, no. 1, pp. 210-225, 2012.

[6] P. Van Mieghem, J. Omic, and R. Kooij, "Virus spread in networks," IEEE/ACM Transactions on Networking, vol. 17, no. 1, pp. 1-14, 2009.

[7] Y. Wang, F. R. Yu, H. Tang, and M. Huang, "A mean field game theoretic approach for security enhancements in mobile ad hoc networks," IEEE Transactions on wireless communications, vol. 13, no. 3, pp. 1616-1627, 2014.

[8] J. Arabneydi and A. G. Aghdam, "Optimal dynamic pricing for binary demands in smart grids: A fair and privacy-preserving strategy," in Proceedings of American Control Conference, 2018, pp. 5368-5373.

[9] O. Madani, S. Hanks, and A. Condon, "On the undecidability of probabilistic planning and related stochastic optimization problems," Artificial Intelligence, vol. 147, no. 1, pp. 5-34, 2003.

[10] C. H. Papadimitriou and J. N. Tsitsiklis, "The complexity of Markov decision processes," Mathematics of Operations Research, vol. 12, no. 3, pp. 441-450, 1987.

[11] N. Meuleau, K.-E. Kim, L. P. Kaelbling, and A. R. Cassandra, "Solving POMDPs by searching the space of finite policies," in Proceedings of the 15th Conference on Uncertainty in Artificial Intelligence, pp. 417-426, 1999. 
[12] W. S. Lovejoy, "Computationally feasible bounds for partially observed Markov decision processes," Operations Research, vol. 39, no. 1, pp. 162-175, 1991.

[13] R. D. Smallwood and E. J. Sondik, "The optimal control of partially observable Markov processes over a finite horizon," Operations Research, vol. 21, no. 5, pp. 10711088, 1973.

[14] M. L. Littman, "The witness algorithm: Solving partially observable Markov decision processes," Technical report, Brown University, 1994.

[15] L. P. Kaelbling, M. L. Littman, and A. R. Cassandra, "Planning and acting in partially observable stochastic domains," Artificial intelligence, vol. 101, no. 1-2, pp. 99-134, 1998.

[16] G. Shani, J. Pineau, and R. Kaplow, "A survey of pointbased POMDP solvers," Autonomous Agents and MultiAgent Systems, vol. 27, no. 1, pp. 1-51, 2013.

[17] E. A. Hansen, "Finite-memory control of partially observable systems," in Proceedings of the 14th Conference on Uncertainty in Artificial Intelligence, pp. 211219, 1998.

[18] V. Krishnamurthy, Partially Observed Markov Decision Processes. Cambridge University Press, 2016.

[19] A. R. Cassandra, "A survey of POMDP applications," Working Notes of AAAI 1998 Fall Symposium on Planning with Partially Observable Markov Decision Processes, pp. 17-24, 1998.

[20] J. Arabneydi, "New concepts in team theory: Mean field teams and reinforcement learning," Ph.D. dissertation, Department of Electrical and Computer Engineering, McGill University, Montreal, Canada, 2016.

[21] J. Arabneydi and A. Mahajan, "Reinforcement learning in decentralized stochastic control systems with partial history sharing," in Proceedings of American Control Conference, 2015, pp. 5449-5456.

[22] Y. Bar-Shalom and E. Tse, "Dual effect, certainty equivalence, and separation in stochastic control," IEEE Transactions on Automatic Control, vol. 19, no. 5, pp. 494-500, 1974.

[23] F. R. Chung, Spectral graph theory. American Mathematical Society, Rhode Island, USA, 1997, vol. 92.

[24] Y. S. Chow and H. Teicher, Probability theory: independence, interchangeability, martingales. Springer Science \& Business Media, 2012.

[25] D. P. Bertsekas, Dynamic programming and optimal control. Athena Scientific, 4th Edition, 2012.

[26] R. Toral and P. Colet, Stochastic numerical methods: an introduction for students and scientists. John Wiley \& Sons, 2014.

[27] W. Wu and A. Arapostathis, "Optimal sensor querying: General markovian and LQG models with controlled observations," IEEE Transactions on Automatic Control, vol. 53, no. 6, pp. 1392-1405, 2008.

[28] J. A. Fuemmeler, G. K. Atia, and V. V. Veeravalli, "Sleep control for tracking in sensor networks," IEEE Transactions on Signal Processing, vol. 59, no. 9, pp. 4354-4366, 2011.
[29] J. Arabneydi and A. G. Aghdam, "A certainty equivalence result in team-optimal control of mean-field coupled Markov chains," in Proceedings of the 56th IEEE Conference on Decision and Control, 2017, pp. 31253130 .

[30] P. Diaconis and D. Freedman, "De Finetti's theorem for Markov chains," The Annals of Probability, JSTOR, pp. 115-130, 1980.

[31] G. M. Lipsa and N. C. Martins, "Remote state estimation with communication costs for first-order LTI systems," IEEE Transactions on Automatic Control, vol. 56, no. 9, pp. 2013-2025, 2011.

[32] A. Nayyar, T. Başar, D. Teneketzis, and V. V. Veeravalli, "Optimal strategies for communication and remote estimation with an energy harvesting sensor," IEEE Transactions on Automatic Control, vol. 58, no. 9, pp. 2246-2260, 2013.

[33] J. P. Hespanha, P. Naghshtabrizi, and Y. Xu, "A survey of recent results in networked control systems," in Proceedings of the IEEE, vol. 95, no. 1, pp. 138-162, 2007.

[34] D. S. Alberts and D. S. Papp, "The information age: An anthology on its impact and consequences," Part 1: The Information, Communication Revolution, Office of the Assistant Secretary of Defense Washington DC Command, and control research program, Tech. Rep., 1997.

[35] S. Kaul, R. Yates, and M. Gruteser, "Real-time status: How often should one update?" in Proceedings of IEEE INFOCOM, pp. 2731-2735, 2012.

[36] I. Kadota, A. Sinha, E. Uysal-Biyikoglu, R. Singh, and E. Modiano, "Scheduling policies for minimizing age of information in broadcast wireless networks," IEEE/ACM Transactions on Networking, vol. 26, no. 6, pp. 2637-2650, 2018.

[37] L. Meier, J. Peschon, and R. Dressler, "Optimal control of measurement subsystems," IEEE Transactions on Automatic Control, vol. 12, no. 5, pp. 528-536, 1967.

[38] J. Arabneydi and A. G. Aghdam, "Near-optimal design for fault-tolerant systems with homogeneous components under incomplete information," in Proceedings of the 61st IEEE International Midwest Symposium on Circuits and Systems, 2018, pp. 809-812.

[39] M. Baharloo, J. Arabneydi, and A. G. Aghdam, "Minmax mean-field team approach for a leader-follower network: A saddle-point strategy," IEEE Control Systems Letters, vol. 4, no. 1, pp. 121-126, 2019.

[40] J. Arabneydi and A. G. Aghdam, "A mean-field team approach to minimize the spread of infection in a network," in Proceedings of American Control Conference, 2019, pp. 2747-2752.

[41] J. N. Tsistsiklis, "Asynchronous stochastic approximation and Q-learning," Machine Learning, vol. 16, pp. 185-202, 1994. 


\section{APPENDIX A}

Proof of THEOREM 1

From (1) and (2), it follows that for any $s^{\prime} \in \mathcal{S}, t \in \mathbb{N}$,

$$
\begin{aligned}
n \cdot m_{t+1}\left(s^{\prime}\right) & =\sum_{i=1}^{n} \mathbb{1}\left(s_{t+1}^{i}=s^{\prime}\right)=\sum_{i=1}^{n} \mathbb{1}\left(f\left(s_{t}^{i}, m_{t}, w_{t}^{i}\right)=s^{\prime}\right) \\
& =\sum_{s \in \mathcal{S}} \sum_{i=1}^{n} \mathbb{1}\left(s_{t}^{i}=s\right) \mathbb{1}\left(f\left(s, m_{t}, w_{t}^{i}\right)=s^{\prime}\right) .
\end{aligned}
$$

For every state $s \in \mathcal{S}$, the inner sum in equation $(33)$ has $n(1-$ $\left.m_{t}(s)\right)$ zero terms because there are only $n m_{t}(s)$ terms with state $s$ according to the definition of empirical distribution (11). These $n m_{t}(s)$ possibly non-zero terms are independent binary random variables, according to Assumption 2 , with the success probability $\mathbb{P}\left(\mathbb{1}\left(f\left(s, m_{t}, w_{t}^{i}\right)=s^{\prime}\right)=1\right)=\sum_{w \in \mathcal{W}} P_{W}\left(w_{t}^{i}=\right.$ $w) \times \mathbb{1}\left(f\left(s, m_{t}, w\right)=s^{\prime}\right)=T\left(s^{\prime}, s, m_{t}\right)$. Let $\phi_{m(s)}\left(s^{\prime}, s, m_{t}\right)$ denote the probability distribution function of the sum of these $n m_{t}(s)$ Bernoulli random variables, which is a binomial distribution with $n m_{t}(s)$ trials and success probability $T\left(s^{\prime}, s, m_{t}\right)$. Now, the probability distribution function of $n m_{t+1}\left(s^{\prime}\right)$ is the probability distribution function of the outer sum in equation (33), consisting of $|\mathcal{S}|$ independent random variables (due to Assumption [2), each of which has the probability distribution function $\phi_{m(s)}\left(s^{\prime}, s, m_{t}\right)$. Therefore, the probability distribution of $n m_{t+1}\left(s^{\prime}\right)$ can be expressed as the convolution of the probability distribution functions $\phi_{m(s)}\left(s^{\prime}, s, m_{t}\right)$ over space $\mathcal{S}$, denoted by $\bar{\phi}\left(s^{\prime}, m_{t}\right)$.

\section{APPENDiX B}

PROOF OF LEMMA 2

One can write:

$$
\begin{gathered}
\mathbb{P}\left(o_{t+1} \mid x_{1: t}, y_{1: t}, a_{1: t}\right)=\sum_{m^{\prime}} \mathbb{P}\left(m_{t+1}=m^{\prime} \mid x_{1: t}, y_{1: t}, a_{1: t}\right) \\
\times \mathbb{P}\left(o_{t+1} \mid m_{t+1}=m^{\prime}, x_{1: t}, y_{1: t}, a_{1: t}\right) .
\end{gathered}
$$

From (3) and (4), the multiplicand in the right-hand side of equation (34) is given by:

$$
\begin{array}{r}
\mathbb{P}\left(o_{t+1} \mid m_{t+1}=m^{\prime}, x_{1: t}, y_{1: t}, a_{1: t}\right)=a_{t} q \mathbb{1}\left(o_{t+1}=m^{\prime}\right) \\
+\left(1-a_{t} q\right) \mathbb{1}\left(o_{t+1}=\mathrm{blank}\right) .
\end{array}
$$

The multiplier in the right-hand side of equation (34), on the other hand, is:

$$
\begin{aligned}
& \mathbb{P}\left(m_{t+1}=m^{\prime} \mid x_{1: t}, y_{1: t}, a_{1: t}\right) \\
& =\sum_{m \in \mathcal{M}(n)} \mathbb{P}\left(m_{t+1}=m^{\prime} \mid m_{t}=m, x_{1: t}, y_{1: t}, a_{1: t}\right) \times \\
& \mathbb{P}\left(m_{t}=m \mid x_{1: t}, y_{1: t}, a_{1: t}\right) \\
& \stackrel{(a)}{=} \sum_{m \in \mathcal{M}(n)} \mathbb{P}\left(m_{t+1}=m^{\prime} \mid m_{t}=m\right) \times \\
& \frac{\mathbb{1}\left(a_{t}=g_{t}\left(o_{1: t}, a_{1: t-1}\right)\right) \mathbb{P}\left(m_{t}=m \mid x_{1: t}, y_{1: t}, a_{1: t-1}\right)}{\sum_{\tilde{m}} \mathbb{1}\left(a_{t}=g_{t}\left(o_{1: t}, a_{1: t-1}\right)\right) \mathbb{P}\left(m_{t}=\tilde{m} \mid x_{1: t}, y_{1: t}, a_{1: t-1}\right)} \\
& \stackrel{(b)}{=} \sum_{m \in \mathcal{M}(n)} T_{\mathrm{m}}\left(m^{\prime}, m\right) \cdot T_{\mathrm{m}}^{y_{t}}\left(m, x_{t}\right)=T_{\mathrm{m}}^{y_{t}+1}\left(o_{t+1}, x_{t}\right) \text {, }
\end{aligned}
$$

where $(a)$ follows from Proposition 11, the fact that $\mathbf{w}_{t}$ is independent of the information up to time $t$, i.e. $\left(o_{1: t}, a_{1: t}, x_{1: t}, y_{1: t}\right)$, as well as Bayes' rule, and (b) follows from (15) and (18). The proof follows from (34)- (35).

\section{APPENDIX C \\ PROOF OF LEMMA 3}

To prove the lemma, it is noted that by definition:

$$
\begin{aligned}
& \mathbb{E}\left[c\left(m_{t}, \hat{m}_{t}, a_{t}\right) \mid o_{1: t}, a_{1: t}\right] \\
& =\sum_{m, \hat{m} \in \mathcal{M}(n)} c\left(m, \hat{m}, a_{t}\right) \mathbb{P}\left(m_{t}=m, \hat{m}_{t}=\hat{m} \mid o_{1: t}, a_{1: t}\right) \\
& \stackrel{(a)}{=} \sum_{m, \hat{m} \in \mathcal{M}(n)} c\left(m, \hat{m}, a_{t}\right) \mathbb{1}\left(\hat{m}=h\left(\mathbb{P}\left(m_{t}=m \mid o_{1: t}, a_{1: t-1}\right)\right)\right) \\
& \times \frac{\mathbb{1}\left(a_{t}=g_{t}\left(o_{1: t}, a_{1: t-1}\right)\right) \mathbb{P}\left(m_{t}=m \mid o_{1: t}, a_{1: t-1}\right)}{\sum_{\tilde{m} \in \mathcal{M}(n)} \mathbb{1}\left(a_{t}=g_{t}\left(o_{1: t}, a_{1: t-1}\right)\right) \mathbb{P}\left(m_{t}=\tilde{m} \mid o_{1: t}, a_{1: t-1}\right)} \\
& \stackrel{(b)}{=} \sum_{m, \hat{m} \in \mathcal{M}(n)} c\left(m, \hat{m}, a_{t}\right) \mathbb{1}\left(\hat{m}=h\left(T_{\mathrm{m}}^{y_{t}}\left(m, x_{t}\right)\right)\right) T_{\mathrm{m}}^{y_{t}}\left(m, x_{t}\right),
\end{aligned}
$$

where (a) follows from (6) as well as Bayes' rule, and $(b)$ follows from 18 .

\section{APPENDIX D \\ PROOF OF THEOREM 4}

The proof consists of two parts. In the first part, we define a "virtual" finite-state Markov decision process (MDP), and in the second part, we show that the optimal solution of this MDP is an $\varepsilon$-optimal solution of Problem 1 .

Part 1: For any $t \in \mathbb{N}$ and finite $k \in \mathbb{N}$, define a so called "virtual" finite-state MDP with state $\left(\tilde{x}_{t}, \tilde{y}_{t}\right) \in \mathcal{M}(n) \times \mathbb{N}_{k}^{*}$ and action $\tilde{a}_{t} \in \mathcal{A}$, as well as the initial state $\left(\tilde{x}_{1}, \tilde{y}_{1}\right)=\left(x_{1}, y_{1}\right)=$ $\left(m_{1}, 0\right)$. At time $t \in \mathbb{N}$, state $\left(\tilde{x}_{t}, \tilde{y}_{t}\right)$ evolves according to function $\tilde{f}: \mathcal{M}(n) \times \mathbb{N}_{k}^{*} \times \mathcal{O} \rightarrow \mathcal{M}(n) \times \mathbb{N}_{k}^{*}$ as $\left(\tilde{x}_{t+1}, \tilde{y}_{t+1}\right)=$ $\tilde{f}\left(\tilde{x}_{t}, \tilde{y}_{t}, \tilde{o}_{t+1}\right)$ such that

$$
\tilde{f}\left(\tilde{x}_{t}, \tilde{y}_{t}, \tilde{o}_{t+1}\right):= \begin{cases}\left(\tilde{x}_{t}, \tilde{y}_{t}+1\right), & \tilde{o}_{t+1}=\mathrm{blank}, \tilde{y}_{t}<k, \\ \left(m^{*}, 0\right), & \tilde{o}_{t+1}=\mathrm{blank}, \tilde{y}_{t}=k, \\ \left(\tilde{o}_{t+1}, 0\right), & \tilde{o}_{t+1} \neq \mathrm{blank},\end{cases}
$$

where $\tilde{o}_{t+1} \in \mathcal{O}$ is a noise process and $m^{*} \in \mathcal{M}(n)$ is an arbitrary empirical distribution. The probability distribution of the noise $\tilde{o}_{t+1}$ is identical to that of the observation in the original model $[19]$, i.e., $\mathbb{P}\left(\tilde{o}_{t+1} \mid \tilde{x}_{1: t}, \tilde{y}_{1: t}, \tilde{a}_{1: t}\right)=$ $\left(1-\tilde{a}_{t} q\right) \mathbb{1}\left(\tilde{o}_{t+1}=\right.$ blank $)+\tilde{a}_{t} q T_{\mathrm{m}}^{\tilde{y}_{t}+1}\left(\tilde{o}_{t+1}, \tilde{x}_{t}\right) \mathbb{1}\left(\tilde{o}_{t+1} \neq\right.$ blank). The per-step cost of the virtual model introduced above is the restriction function $\hat{c}$ given by 20, over space $\mathcal{M}(n) \times \mathbb{N}_{k}^{*} \times \mathcal{A}$, i.e., at time $t \in \mathbb{N}, \hat{c}\left(\tilde{x}_{t}, \tilde{y}_{t}, \tilde{a}_{t}\right):=$ $\sum_{m \in \mathcal{M}(n)} c\left(m, h\left(T_{\mathrm{m}}^{\tilde{y}_{t}}\left(m, \tilde{x}_{t}\right)\right), a_{t}\right) T_{\mathrm{m}}^{\tilde{y}_{t}}\left(m, \tilde{x}_{t}\right)$. The strategy of the virtual model is given by $\tilde{a}_{t}=\tilde{g}_{t}\left(\tilde{x}_{1: t}, \tilde{y}_{1: t}\right)$, and its performance is described by $\tilde{J}(\tilde{g})=\mathbb{E}^{\tilde{g}}\left[\sum_{t=1}^{\infty} \gamma^{t-1} \hat{c}\left(\tilde{x}_{t}, \tilde{y}_{t}, \tilde{a}_{t}\right)\right]$. From the standard results in Markov decision theory [25], the optimal solution of the virtual model is obtained by solving the following Bellman equation for any $\tilde{x} \in \mathcal{M}(n), \tilde{y} \in \mathbb{N}_{k}^{*}$ :

$$
\tilde{V}_{k}(\tilde{x}, \tilde{y})=\min _{\tilde{a} \in \mathcal{A}}\left(\hat{c}(\tilde{x}, \tilde{y}, \tilde{a})+\gamma \mathbb{E}\left[\tilde{V}_{k}(\tilde{f}(\tilde{x}, \tilde{y}, \tilde{o}))\right]\right),
$$

where the above expectation is taken over all noises $\tilde{o} \in \mathcal{O}$. 
Part 2: Let $J^{*}$ be the performance under the optimal solution of Bellman equation (21) and $\tilde{J}^{*}$ be the performance under the optimal solution of Bellman equation (37). We compute an upper bound on the relative distance between $J^{*}$ and $\tilde{J}^{*}$, i.e. $\left|J^{*}-\tilde{J}^{*}\right|$, as follows:

$$
\begin{aligned}
& \left|\min _{g} \mathbb{E}^{g} \sum_{t=1}^{\infty} \gamma^{t-1} \hat{c}\left(x_{t}, y_{t}, a_{t}\right)-\min _{\tilde{g}} \mathbb{E}^{\tilde{g}} \sum_{t=1}^{\infty} \gamma^{t-1} \hat{c}\left(\tilde{x}_{t}, \tilde{y}_{t}, \tilde{a}_{t}\right)\right| \\
& =\mid \min _{g} \mathbb{E}^{g}\left[\sum_{t=1}^{k} \gamma^{t-1} \hat{c}\left(x_{t}, y_{t}, a_{t}\right)+\sum_{t=k+1}^{\infty} \gamma^{t-1} \hat{c}\left(x_{t}, y_{t}, a_{t}\right)\right] \\
& \quad-\min _{\tilde{g}} \mathbb{E}^{\tilde{g}}\left[\sum_{t=1}^{k} \gamma^{t-1} \hat{c}\left(\tilde{x}_{t}, \tilde{y}_{t}, \tilde{a}_{t}\right)-\sum_{t=k+1}^{\infty} \gamma^{t-1} \hat{c}\left(\tilde{x}_{t}, \tilde{y}_{t}, \tilde{a}_{t}\right)\right] \mid \\
& \stackrel{(a)}{\leq} \frac{2 \gamma^{k} c_{\max }}{1-\gamma},
\end{aligned}
$$

where $(a)$ follows from the triangle inequality, the fact that $c_{\max }$ is an upper bound for the per-step cost, and that the minimization of the expected cost of the original model and that of the virtual model up to time $k$ are essentially the same, as both models start from the same initial state $\left(m_{1}, 0\right)$, follow the same dynamics, and incur the same cost up to time $k$. Finally, it is concluded from Parts 1 and 2 that when $k$ is sufficiently large such that $\frac{2 \gamma^{k} c_{\max }}{1-\gamma} \leq \varepsilon$, the optimal solution of the Bellman equation 37) is an $\varepsilon$-optimal solution of Problem 1 . The proof is completed by incorporating equation (36) in the Bellman equation (37).

\section{APPENDIX E \\ PROOF OF THEOREM 5}

Let $\tilde{V}_{k}^{*}: \mathcal{M}(n) \times \mathbb{N}_{k}^{*} \rightarrow \mathbb{R}_{\geq 0}$ be the optimal value function satisfying 37). Define function $Q^{*}: \mathcal{M}(n) \times \mathbb{N}_{k}^{*} \times \mathcal{A} \rightarrow \mathbb{R}_{\geq 0}$ such that for every $x \in \mathcal{M}(n), y \in \mathbb{N}_{k}^{*}, a \in \mathcal{A}$,

$$
Q^{*}(x, y, a):=\hat{c}(x, y, a)+\gamma \sum_{o \in \mathcal{O}} \mathbb{P}(o \mid x, y, a) \tilde{V}_{k}^{*}(\tilde{f}(x, y, o)) .
$$

It follows from equations (37) and 38 that $\tilde{V}_{k}^{*}(x, y)=$ $\min _{a \in \mathcal{A}} Q^{*}(x, y, a)$ for every $x \in \mathcal{M}(n)$ and $y \in \mathbb{N}_{k}^{*}$, i.e.,

$$
Q^{*}(x, y, a)=\hat{c}(x, y, a)+\gamma \sum_{o \in \mathcal{O}} \mathbb{P}(o \mid x, y, a) \min _{a^{\prime} \in \mathcal{A}} Q^{*}\left(\tilde{f}(x, y, o), a^{\prime}\right) \text {. }
$$

According to Theorem 4 and equations (37) and (38), any $\operatorname{argmin}_{a \in \mathcal{A}} Q^{*}(x, y, a)$ is an argmin for the Bellman equation (22). Now, rewrite equation (39) as $Q^{*}(x, y, a)=c^{\prime}+$ $\gamma \min _{a^{\prime} \in \mathcal{A}} Q^{*}\left(\tilde{f}(x, y, o), a^{\prime}\right)+n(x, y, o)$, where $c^{\prime}$ denotes the instantaneous cost at state $(x, y)$ and action $a$, and the random variable $n(x, y, o)$ is defined by

$$
\begin{aligned}
n(x, y, o):= & -c^{\prime}+\hat{c}(x, y, a)-\gamma \min _{a^{\prime} \in \mathcal{A}} Q^{*}\left(\tilde{f}(x, y, o), a^{\prime}\right) \\
& +\gamma \sum_{o \in \mathcal{O}} \mathbb{P}(o \mid x, y, a) \min _{a^{\prime} \in \mathcal{A}} Q^{*}\left(\tilde{f}(x, y, o), a^{\prime}\right),
\end{aligned}
$$

such that $\mathbb{E}[n(x, y, o) \mid x, y, a]=0$, and $\mathbb{E}\left[n(x, y, o)^{2}\right.$ $x, y, a] \leq 4 c_{\max }^{2}+4\left(\max _{x^{\prime}, y^{\prime}, a^{\prime}} Q^{*}\left(\left(x^{\prime}, y^{\prime}\right), a^{\prime}\right)\right)^{2}$, where $n(x, y, o) \leq 2 c_{\max }+2 \max _{x^{\prime}, y^{\prime}, a^{\prime}} Q^{*}\left(\left(x^{\prime}, y^{\prime}\right), a^{\prime}\right)$. The decision maker can use stochastic approximation theory to approximate the $Q^{*}$-function described by equation (39), because: (i) function $\tilde{f}$ is independent of the model; (ii) the expectation and variance of $n(x, y, o)$ are respectively zero and finite, and (iii) equation (39) is a contraction mapping in the infinity norm due to the discount factor $\gamma<1$, i.e., for any $Q$ and $Q^{\prime}$ :

$$
\left\|F(Q)-F\left(Q^{\prime}\right)\right\| \leq \gamma \max _{x^{\prime}, y^{\prime}, a^{\prime}}\left|Q\left(\left(x^{\prime}, y^{\prime}\right), a^{\prime}\right)-Q^{\prime}\left(\left(x^{\prime}, y^{\prime}\right), a^{\prime}\right)\right|,
$$

where $F$ denotes the function form of equation (39) such that $Q^{*}=F\left(Q^{*}\right)$. Therefore, the following stochastic approximation iteration converges to $Q^{*}$ under standard assumptions in [41. Theorem 4], i.e., for $\tau \in \mathbb{N}$,

$$
\begin{aligned}
& Q_{\tau+1}(x, y, a)=Q_{\tau}(x, y, a)+ \\
& \alpha_{\tau}(x, y, a)\left(c^{\prime}+\gamma \min _{a^{\prime} \in \mathcal{A}} Q_{\tau}\left(\tilde{f}(x, y, o), a^{\prime}\right)-Q_{\tau}(x, y, a)\right) .
\end{aligned}
$$

The proof is completed on noting that the obtained greedy strategy $g_{\varepsilon}^{*}$ is an $\varepsilon$-optimal solution, according to Theorem 4

\section{APPENDIX F \\ ProOF OF THEOREM 6}

The total expected discounted cost for any finite horizon $H \in \mathbb{N}$ under no collection action is given by:

$$
\begin{aligned}
& \underset{t=1}{\mathbb{E}}\left[\sum_{t=1}^{H} \gamma^{t-1} c\left(m_{t}, \hat{m}_{t}, 0\right)\right]_{\leq}^{(a)} \mathbb{E}\left[\sum_{t=1}^{H} K_{c} \gamma^{t-1}\left\|m_{t}-\hat{m}_{t}\right\|\right] \\
& \stackrel{(b)}{\leq} K_{c} \mathbb{E}\left[\left\|m_{1}-\hat{m}_{1}\right\|\right]+K_{c} \sum_{t=2}^{H} \gamma^{t-1} \mathbb{E}\left[K_{p}^{t-1}\left\|m_{1}-\hat{m}_{1}\right\|\right. \\
& \left.\quad+\left(\sum_{\tau=1}^{t-1} K_{p}^{\tau-1}\right) \mathcal{O}\left(\frac{1}{\sqrt{n}}\right)\right] \\
& \stackrel{(c)}{=} K_{c} \sum_{t=2}^{H} \gamma^{t-1}\left(\sum_{\tau=1}^{t-1} K_{p}^{\tau-1}\right) \mathcal{O}\left(\frac{1}{\sqrt{n}}\right) \\
& \stackrel{(d)}{=} K_{c} \sum_{t=2}^{H} \gamma^{H-t+1}\left(\sum_{\tau=1}^{t-1}\left(\gamma K_{p}\right)^{\tau-1}\right) \mathcal{O}\left(\frac{1}{\sqrt{n}}\right) \\
& \stackrel{(e)}{\leq} K_{c} \sum_{t=2}^{H} \gamma^{H-t+1}\left(\sum_{\tau=1}^{H-1}\left(\gamma K_{p}\right)^{\tau-1}\right) \mathcal{O}\left(\frac{1}{\sqrt{n}}\right) \\
& =K_{c}\left(\sum_{t=2}^{H} \gamma^{H-t+1}\right)\left(\sum_{\tau=1}^{H-1}\left(\gamma K_{p}\right)^{\tau-1}\right) \mathcal{O}\left(\frac{1}{\sqrt{n}}\right) \\
& \stackrel{(f)}{=} K_{c} \times \frac{\gamma\left(1-\gamma \gamma^{H-1}\right)}{1-\gamma} \times \frac{1-\left(\gamma K_{p}\right)^{H-1}}{1-\gamma K_{p}} \times \mathcal{O}\left(\frac{1}{\sqrt{n}}\right), \\
&
\end{aligned}
$$

where $(a)$ follows from Assumption 3 and the monotonicity of the expectation operator; $(b)$ follows from Lemma 5 (by applying it recursively); (c) follows from the fact that $m_{1}=$ $\hat{m}_{1} ;(d)$ rearranges the terms; $(e)$ follows from the fact that $\gamma K_{p}$ is non-negative along with the inequality $t \leq H$, and ( $f$ ) follows from Assumption 4 The proof is completed by tending horizon $H$ to $\infty$.

\section{APPENDIX G \\ PROOF OF THEOREM 7}

Given any strategy $g$, the information set $\left\{o_{1: t}, a_{1: t-1}\right\}$ can be equivalently expressed by the set $\left\{x_{1: t}, y_{1: t}\right\}$, according 
to (5) and (17). Hence, the generic estimator $h$ can be represented by a strategy-dependent estimator $\hat{m}_{t}=h_{t}^{g}\left(x_{1: t}, y_{1: t}\right)$. From (9) and the definition of the set $\left\{x_{1: t}, y_{1: t}\right\}$, one has:

$$
m_{t}=A^{y_{t}} x_{t}+\mathbb{1}\left(y_{t}>0\right) \sum_{\tau=1}^{y_{t}} A^{\tau-1} \bar{w}_{t-\tau} .
$$

Since local noises from time $t-y_{t}$ to time $t$ have zero mean, and are mutually independent over time (and so is $\bar{w}_{t-y_{t}: t}$ ), the per-step cost function can be described as follows:

$$
\begin{aligned}
& \mathbb{E}\left[\left(m_{t}-\hat{m}_{t}\right)^{\top}\left(m_{t}-\hat{m}_{t}\right) z\left(a_{t}\right)+\ell\left(m_{t}, a_{t}\right) \mid x_{1: t}, y_{1: t}, g_{1: t}\right] \\
& =\mathbb{E}\left[\left(A^{y_{t}} x_{t}-h_{t}^{g}\left(x_{1: t}, y_{1: t}\right)\right)^{\top}\left(A^{y_{t}} x_{t}-h_{t}^{g}\left(x_{1: t}, y_{1: t}\right)\right]\right. \\
& \times z\left(g_{t}\left(x_{1: t}, y_{1: t}\right)\right) \\
& +\mathbb{1}\left(y_{t}>0\right) z\left(g_{t}\left(x_{1: t}, y_{1: t}\right)\right) \sum_{\tau=1}^{y_{t}} \mathbb{E}\left[\left(\bar{w}_{t-\tau}\right)^{\top}\left(A^{\tau-1}\right)^{\top} A^{\tau-1} \bar{w}_{t-\tau}\right] \\
& +\mathbb{E}\left[\ell\left(A^{y_{t}} x_{t}+\mathbb{1}\left(y_{t}>0\right) \sum_{\tau=1}^{y_{t}} A^{\tau-1} \bar{w}_{t-\tau}, g_{t}\left(x_{1: t}, y_{1: t}\right)\right)\right] .
\end{aligned}
$$

Therefore, for any control horizon $H$, any sample path $\left\{x_{1: H}, y_{1: H}\right\}$, and any strategy $g_{1: H}$, the first term in the right-hand side of the above equation is the only term that is affected by the choice of $h^{g}$, which yields the unique minimizer $h_{t}^{g}\left(x_{1: t}, y_{1: t}\right)=A^{y_{t}} x_{t}$. Note that the structure of this estimator is independent of the probability distribution of the underlying random variables, strategy $g$, and the order of dynamics, and follows the update rule $\hat{m}_{t+1}=A^{y_{t+1}} x_{t+1}$, i.e., equation (27). This estimator has the same structure as the minimum mean-square estimator $\mathbb{E}\left[m_{t} \mid o_{1: t}, a_{1: t-1}\right]$. Hence,

$\hat{m}_{t+1}= \begin{cases}x_{t+1}=o_{t+1}, & o_{t+1} \neq \mathrm{blank}\left(\text { i.e., } y_{t+1}=0\right), \\ A^{1+y_{t}} x_{t}=A \hat{m}_{t}, & o_{t+1}=\mathrm{blank}\left(\text { i.e., } y_{t+1}>0\right) .\end{cases}$

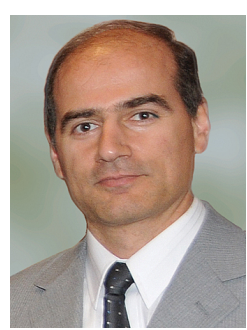

Amir G. Aghdam received the Ph.D. degree in electrical and computer engineering from the University of Toronto, Toronto, ON, Canada, in 2000. $\mathrm{He}$ is currently a Professor in the Department of Electrical and Computer Engineering at Concordia University, Montreal, QC, Canada. He was a Visiting Scholar at Harvard University in fall 2015, and was an Associate with the Harvard John A. Paulson School of Engineering and Applied Sciences from September 2015 to December 2016. His research interests include multi-agent networks, distributed control, optimization and sampled-data systems. He is a member of Professional Engineers Ontario, chair of the Conference Editorial Board of IEEE Control Systems Society, Editor-in-Chief of the IEEE Systems Journal, and was an Associate Editor of the IEEE Transactions on Control Systems Technology and the Canadian Journal of Electrical and Computer Engineering. He has been a member of the Technical Program Committee of a number of conferences, including the IEEE Conference on Systems, Man and Cybernetics (IEEE SMC)Inline image and the IEEE Multiconference on Systems and Control (IEEE MSC). He was a member of the Review Committee for the Italian Research and University Evaluation Agency (ANVUR) for 2012-2013, and a member of the Natural Sciences and Engineering Research Council of Canada (NSERC) ECE Evaluation Group for 2014-2016. He is a recipient of the 2009 IEEE MGA Achievement Award, the 2011 IEEE Canada J. J. Archambault Eastern Canada Merit Award, and the 2020 IEEE Canada J. M. Ham Outstanding Engineering Educator Award. He was the 2014-2015 President of IEEE Canada and Director (Region 7), IEEE, Inc., and was also a member of the IEEE Awards Board for this period. Dr. Aghdam was a member of the IEEE Medal of Honor Committee for 2017-2019, and IEEE MGA Awards and Recognition Committee for 2017-2018, and is currently the Vice-Chair of the IEEE Medals Council.

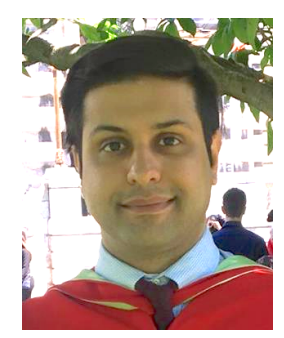

Jalal Arabneydi received the Ph.D. degree in Electrical and Computer Engineering from McGill University, Montreal, Canada in 2017. He is currently a postdoctoral fellow at Concordia University. $\mathrm{He}$ was the recipient of the best student paper award at the 53rd Conference on Decision and Control (CDC), 2014. His principal research interests include stochastic control, robust optimization, game theory, large-scale system, multi-agent reinforcement learning with applications in complex networks including smart grids, swarm robotics, and finance. His current research interest is focused on what he calls deep planning, which bridges decision making theory and artificial intelligence. The ultimate goal is to define proper mathematical tools and solution concepts in order to develop large-scale decision-making algorithms that work under imperfect information and incomplete knowledge with analytical performance guarantees. 